\title{
Comparative genomic analysis of the principal Cryptosporidium species that infect humans
}

\author{
Laura M. Arias-Agudelo ${ }^{1}$, Gisela Garcia-Montoya ${ }^{1}$, Felipe Cabarcas ${ }^{1,2}$, Ana L. Galvan-Diaz ${ }^{3}$, Juan F. Alzate ${ }^{\text {Corresp. } 1}$ \\ ${ }^{1}$ Centro Nacional de Secuenciación Genómica - CNSG, Sede de Investigación Universitaria - SIU, Departamento de Microbiología y Parasitología, Facultad \\ de Medicina, Universidad de Antioquia, Medellin, Antioquia, Colombia \\ 2 Grupo SISTEMIC, Departamento de Ingeniería Electrónica, Facultad de Ingeniería, Universidad de Antioquia, Medellin, Antioquia, Colombia \\ 3 Environmental Microbiology Group, School of Microbiology, Universidad de Antioquia, Medellin, Antioquia, Colombia \\ Corresponding Author: Juan F. Alzate \\ Email address: jfernando.alzate@udea.edu.co
}

Cryptosporidium parasites are ubiquitous and can infect a broad range of vertebrates and are considered the most frequent protozoa associated with waterborne parasitic outbreaks. The intestine is the target of three of the species most frequently found in humans: C. hominis, C. parvum, and. C. meleagridis. Despite the recent advance in genome sequencing projects for this apicomplexan, a broad genomic comparison including the three species most prevalent in humans have not been published so far. In this work, we downloaded raw NGS data, assembled it under normalized conditions, and compared 23 publicly available genomes of $C$. hominis, $C$. parvum, and $C$. meleagridis. Although few genomes showed highly fragmented assemblies, most of them had less than 500 scaffolds and mean coverage that ranged between 35X and 511X. Synonymous single nucleotide variants were the most common in $C$. hominis and $C$. meleagridis, while in $C$. parvum, they accounted for around $50 \%$ of the SNV observed. Furthermore, deleterious nucleotide substitutions common to all three species were more common in genes associated with DNA repair, recombination, and chromosome-associated proteins. Indel events were observed in the 23 studied isolates that spanned up to 500 bases. The highest number of deletions was observed in C. meleagridis, followed by $C$. hominis, with more than 60 species-specific deletions found in some isolates of these two species. Although several genes with indel events have been partially annotated, most of them remain to encode uncharacterized proteins. 
1 Comparative genomic analysis of the principal Cryptosporidium species that infect humans

2 Laura M. Arias-Agudelo ${ }^{1}$, Gisela Garcia-Montoya ${ }^{1}$, Felipe Cabarcas ${ }^{1,2}$, Ana L. Galvan-Diaz ${ }^{3}$ \& 3 Juan F. Alzate ${ }^{1 *}$.

4

${ }^{1}$ Centro Nacional de Secuenciación Genómica - CNSG, Sede de Investigación Universitaria SIU. Departamento de Microbiología y Parasitología, Facultad de Medicina, Universidad de Antioquia. Medellín, Antioquia, Colombia

${ }^{2}$ Grupo SISTEMIC, Departamento de Ingeniería Electrónica, Facultad de Ingeniería, Universidad de Antioquia. Medellín, Antioquia, Colombia

3 Environmental Microbiology Group, School of Microbiology, Universidad de Antioquia, Medellin, Antioquia, Colombia

12

Corresponding Author:

Juan F. Alzate. Carrera 53 \# 61 - 30, Medellín, Antioquia, 050010, Colombia Email address: jfernando.alzate@udea.edu.co

16

\begin{abstract}
Cryptosporidium parasites are ubiquitous and can infect a broad range of vertebrates and are considered the most frequent protozoa associated with waterborne parasitic outbreaks. The intestine is the target of three of the species most frequently found in humans: C. hominis, $C$. parvum, and. C. meleagridis. Despite the recent advance in genome sequencing projects for this apicomplexan, a broad genomic comparison including the three species most prevalent in humans have not been published so far. In this work, we downloaded raw NGS data, assembled it under normalized conditions, and compared 23 publicly available genomes of $C$. hominis, $C$. parvum, and $C$. meleagridis. Although few genomes showed highly fragmented assemblies, most of them had less than 500 scaffolds and mean coverage that ranged between $35 \mathrm{X}$ and $511 \mathrm{X}$. Synonymous single nucleotide variants were the most common in $C$. hominis and $C$. meleagridis, while in C. parvum, they accounted for around 50\% of the SNV observed. Furthermore, deleterious nucleotide substitutions common to all three species were more common in genes associated with DNA repair, recombination, and chromosome-associated proteins. Indel events were observed in the 23 studied isolates that spanned up to 500 bases. The highest number of deletions was observed in $C$. meleagridis, followed by $C$. hominis, with more than 60 speciesspecific deletions found in some isolates of these two species. Although several genes with indel events have been partially annotated, most of them remain to encode uncharacterized proteins. Keywords: Cryptosporidium, molecular biology, computational biology, high-throughput nucleotide sequencing, NGS, de novo genome assembly, whole-genome sequencing, genomics, genetic variation, comparative genomics.

\section{Introduction}

Cryptosporidium is a ubiquitous enteric apicomplexan that infects a broad range of vertebrates, including humans and domestic and wild animals (Khan, Shaik \& Grigg, 2017). It is described as an important cause of chronic diarrhea in AIDS and other immunocompromised patients. It is also a cause of death in children under 24 months old, especially in low-income countries (Sow et al., 2016; Troeger et al., 2017). Furthermore, Cryptosporidium is the most frequent protozoa associated with waterborne parasitic outbreaks worldwide (Efstratiou, Ongerth \& Karanis, 2017).
\end{abstract}


46 Cryptosporidium is classified as a gregarine, within its subclass, the Cryptogregaria (Ryan et al., 47 2016). Thus far, there are at least 39 species established, and more than 30 genotypes (Firoozi et 48 al., 2019). Among them, approximately twenty-one have been found in humans. However,

49 Cryptosporidium hominis and Cryptosporidium parvum are responsible for more than $90 \%$ of the 50 reported human infection cases worldwide (Grinberg \& Widmer, 2016; Feng, Ryan \& Xiao, 2018). Other species, occasionally described in humans, including C. meleagridis, $C$. felis, $C$. canis, C. ubiquitum, C. cuniculus, $C$. viatorum, $C$. muris, chipmunk genotype I, $C$. andersoni, $C$. suis, $C$. bovis, horse genotype, $C$. xiaoi, skunk genotype, mink genotype, C. erinacei, $C$. fayeri, C. scrofarum and C. tyzzeri (Feng, Ryan \& Xiao, 2018). These species and genotypes differ significantly in human infectivity, host range, geographic distribution, and virulence (Cama et al., 2007, 2008; Adamu et al., 2014; Feng, Ryan \& Xiao, 2018). Some species, such as $C$. hominis, have very narrow host ranges, mostly restricted to humans, nonhuman primates, and horses, whereas others, such as $C$. parvum, have a broad host range, infecting ruminants, horses, rodents, and other animals besides humans (Ryan, Fayer \& Xiao, 2014). Cryptosporidium meleagridis, which is the third most prevalent species in humans, has been described in mammals and birds (Stensvold et al., 2014). Cryptosporidium molecular phylogeny and evolutionary relationships have been studied through PCR of single or multiple genetic markers (Feng et al., 2007; Xiao, 2010; Xiao \& Feng, 2017; Cunha, Peralta \& Peralta, 2019). Cryptosporidium species identification protocols usually include the amplification and sequencing of the small subunit (SSU) rRNA gene. Additionally, glycoprotein 60 gene (gp60) has been used to study subtypes and intra-species diversity of the genus, leading to the currently accepted classification into gp60 allelic families subtypes (Xiao, 2010). The SSU rDNA gene in Cryptosporidium species does not evolve under a neutral model, and its genetic diversity is restricted to a few polymorphic sites (Sulaiman, Lal \& Xiao, 2002). Additionally, some species appear to evolve much quicker than others, according to the SSU rDNA locus.

Regarding the gp60 gene, its high genetic diversity has been attributed to the action of positive selective pressure. Because it can be exchanged by genetic recombination, its typing information does not always agree with other loci, especially with some $C$. parvum subtype families (Feng, Ryan \& Xiao, 2018). Other loci used in the Cryptosporidium typing include coding genes of actin, $70 \mathrm{kDa}$ heat-shock protein (HSP70), and the Cryptosporidium oocyst wall protein (COWP) (Cunha, Peralta \& Peralta, 2019). Most of these loci do not evolve neutrally, and the rate of evolution may vary among the different species of the genus (Sulaiman, Lal \& Xiao, 2002). According to the above, phylogenetic inference based on analysis of one or a few genetic loci, some under selection pressure, might not reflect the real phylogenetic relationships at the whole genome level (Sulaiman, Lal \& Xiao, 2002; Morris et al., 2019). The growing use of whole-genome shotgun sequencing (WGS) and next-generation sequencing (NGS) in the study of Cryptosporidium spp. is allowing better phylogenetic and comparative genomic analysis within the genus (Widmer \& Sullivan, 2012; Mazurie et al., 2013a; Guo et al., 2015; Isaza et al., 2015; Ifeonu et al., 2016; Beser et al., 2017; Sikora et al., 2017a; Feng et al., 2017; Khan, Shaik \& Grigg, 2017; Gilchrist et al., 2018; Fan, Feng \& Xiao, 2019; Xu et al., 2019; Nader et al., 2019). The first Cryptosporidium genomes were generated in 2004 using capillary sequencing, belonging to the C. parvum (Iowa strain) and C. hominis (TU502 strain) species (Xu et al., 2004; Abrahamsen, 2004). Since then, high-quality (NGS-based) genomes

90 from several subtypes of these species and other human-related species are increasingly available 91 (Widmer \& Sullivan, 2012; Mazurie et al., 2013a; Guo et al., 2015; Beser et al., 2017; Feng et 
92 al., 2017; Sikora et al., 2017a; Gilchrist et al., 2018; Nader et al., 2019; Xu et al., 2019).

93 Comparative analysis shows a remarkable structural and compositional conservation in genome 94 organization among intestinal Cryptosporidium species (Fan, Feng \& Xiao, 2019). The genome size is near $9.0 \mathrm{Mb}$ in length and is arranged into eight chromosomes, with perfect synteny (no evidence of genome rearrangements), extremely compact coding genes, and a low number of gene introns (Fan, Feng \& Xiao, 2019). It has been postulated that the phenotypic differences between Cryptosporidium species could be associated with minor sequence variations (Single nucleotide variants-SNVs and short indels) that can affect expressed proteins or gene regulation patterns (Guo et al., 2015; Feng et al., 2017; Sikora et al., 2017a; Gilchrist et al., 2018; Su et al., 2019; Xu et al., 2019). Nonetheless, several studies have demonstrated events of major insertions and deletions between several species of Cryptosporidium, usually involving members of multicopy gene families under positive selection, such as those located near telomeres, like the MEDLE proteins, insulinase-like proteases, and mucin-type glycoproteins. These genes have been associated with the host-parasite interaction (Guo et al., 2015; Feng et al., 2017; Gilchrist et al., 2018; Xu et al., 2019).

Molecular phylogenetic strategies have been developed to understand the evolutionary relationships between proteins or genes and help to unravel the evolutionary history of the species. Phylogenetic analysis can also give insights into epidemiological, immunological, and evolutionary processes shaping genetic variation in natural populations of the parasite, and may even have the potential to improve future public health measures (Baele et al., 2017; Theys et al., 2019). Although phylogenomics studies on apicomplexan parasites are scarce, in models such as Piroplasmida and Haemosporida, they have been useful in the elucidation of the taxonomy and phylogenetic relationships within these protozoa, through the incorporation of a broad number of taxons and DNA datasets (Cornillot et al., 2012; Lack, Reichard \& Van Den Bussche, 2012; Galen et al., 2018).

Here we used a comprehensive phylogenomic approach to have a better view of the evolutionary relationships of the three most relevant human infecting species of Cryptosporidium protozoan parasites (C. parvum, C. hominis, and C. meleagridis), including genomes with different subtype families. Furthermore, a detailed comparative analysis of the largest indel events detected in these three species is presented, which allowed a more comprehensive view of the gene content differences among these three apicomplexan species.

\section{Methods}

\section{NGS data and assembly}

Read sequences were downloaded from the Sequence Read Archive - SRA of the NCBI public database. Reads of 23 genomic projects of three Cryptosporidium species were included: $C$. parvum, $C$. hominis, and $C$. meleagridis. Four genomes of $C$. meleagridis, ten genomes of $C$. hominis, and nine genomes of $C$. parvum, including the $C$. parvum anthroponotic isolates UKP14 and UKP15 were analyzed. The reads were extended using FLASH v1.2.11 (Magoc \& Salzberg, 2011); then the extended and independent read pairs were assembled with the software SPADES v3.11.1 (Bankevich et al., 2012), with default settings and testing k-mers of 33, 55, 77, 99, and 111 bases; then the descriptive statistics of the assembly were calculated with in house Perl scripts. To avoid any possible contamination of the sequences with other species, the assembled contigs were aligned using BLASTN with the reference genomes (chromosomes) of $C$. hominis UdeA01, C. parvum Iowa II and C. tyzzeri UGA55, downloaded from CryptoDB v43 (Puiu, 2004). The contigs with a Bit score value $\geq 300$ were kept for further analyses. 
138

139

140

141

142

143

144

145

146

147

148

149

150

151

152

153

154

155

156

157

158

159

160

161

162

163

164

165

166

167

168

169

170

171

172

173

174

175

176

177

178

179

180

181

182

183

\section{Sequencing depth analysis}

All the reads were mapped against the C. parvum Iowa II reference genome downloaded from CryptoDB v43 and against the de novo assembled contigs using BWA (Li \& Durbin, 2010) with default options. Then, the Samtools-depth tool (Li et al., 2009) was used to compute the read depth at all positions, with a maximum coverage depth to 1000000 . Finally, the mean coverage was estimated in the Linux terminal with an awk formula.

\section{Single Nucleotide Variants detection}

Detection of SNVs was performed aligning with MUMmer v3 the assembled scaffolds for each isolate with the C. parvum Iowa II reference genome. Then, the MUMmer function show-SNPs, and dnaDIFF tools were used (Delcher et al., 2002).

\section{Phylogenomic analysis}

The phylogenomic analysis was performed based on the SNVs found within the 24 selected Cryptosporidium genomes. A matrix comprising all the SNVs detected was constructed and loaded into the program IQ-TREE v1.6.12 (Trifinopoulos et al., 2016). A maximumlikelihood (ML) tree (Felsenstein, 1981) was built using this phylogenomic inference software. Modelfinder was used to select the best model of evolution under Bayesian Information Criterion (BIC) (Schwarz, 1978). Transversion Model TVM (TVM+F+ASC+R2) was selected as an evolutionary model. Branch support was estimated using 1000 iterations of the ShimodairaHasegawa test (SH -aLRT) (Shimodaira \& Hasegawa, 1999) and 1000 pseudoreplicates of ultrafast bootstrap (Hoang et al., 2018). The presented tree is unrooted, and the longest branch was selected as an arbitrary outgroup. The tree was edited using FigTree v1.4.4 (Andrew Rambaut, 2018).

\section{Identification of SNVs in coding regions}

For the detection of single nucleotide variants in the CDSs, the reads were mapped to the genome of C. parvum Iowa II with the BWA (Burrows-Wheeler Aligner) aligner version 0.7.17 (Li \& Durbin, 2010). Later, the bam and VCF files were generated with SAMtools and BCFtools, respectively $(130,131)$. Only variants with a Phred quality score $\geq 50$ (Holder et al., 2013) were included. Finally, the SAMtools Depth tool was used to calculate the reading depth at all positions, with a maximum coverage depth of $1,000,000$. Subsequently, the mean coverage was estimated with an awk formula. The single nucleotide variants in CDSs were annotated with the effect predictor SIFT4G (sorting intolerant from tolerant) version 3.0 (Vaser et al., 2016).

\section{Identification of insertions and deletions}

Insertions and deletions - indels - were identified with MUMmer aligner (Maximal Unique Matches) version 3.0 (Delcher et al., 2002), as previously described by Isaza et al. (Isaza et al., 2015), excluding those with a length $<50$ nucleotides with the software online Assemblytics (Nattestad \& Schatz, 2016). To perform the functional analyzes, the genome of $C$. parvum Iowa II version 43 deposited in CryptoDB (Puiu, 2004) was used as a reference. Variants were initially detected in coding regions, and then genes with shared and species-exclusive deleterious mutations were identified and annotated.

\section{Identification and annotation of genes with deleterious non-synonymous changes}


184 From the prediction obtained with SIFT4G, genes with deleterious mutations in the eight

185 chromosomes were filtered for each of the genomes. These genes were extracted with the

186 SeqSelect.py script, and enrichment analysis in Gene Ontology terms was performed with the

187 EggNOG mapper program version 1.0 (Huerta-Cepas et al., 2017); and visualization with

188 WEGO (Web Gene Ontology Annotation Plotting) version 2.0 was done (Ye et al., 2018). Then,

189 functional orthology analysis was performed with KEGG on the KAAS server (Ogata et al.,

190 1999), selecting the best hits using a bidirectional strategy - BBH (bi-directional best hit). To

191 detect genes with transmembrane domains, the TMHMM version 2.0 server (Krogh et al., 2001)

192 was used. Prediction of the genes that code for proteins with classical and non-classical secretion

193 was performed with the online servers SIGNALP version 5.0 (Almagro Armenteros et al., 2019)

194 and SECRETOME-P version 2.0 (Bendtsen et al., 2004), respectively.

195

196

197

198

199

200

201

202

203

204

205

206

207

208

209

210

211

212

213

214

215

216

217

218

219

220

221

222

223

224

225

226

227

228

229

\section{Results}

\section{Selected Cryptosporidium genomes}

To carry out a comprehensive genomic comparative analysis, we chose all the NGS projects of the three main species of Cryptosporidium that infect humans (C. hominis, C. parvum, and C. meleagridis), that have raw sequence data available in public databases. A total of 23 genomes were included: ten isolates of Cryptosporidium hominis (UKH1, UKH3, UKH4, UKH5, 37999, TU502_2012, 30976, UdeA01, SWEH2, and SWEH5); nine isolates of C. parvum (UKP2, UKP3, UKP4, UKP5, UKP6, UKP7, UKP8, and the C. parvum anthroponotic isolates UKP14 and UKP15); and four isolates of $C$. meleagridis (UKMEL1, UKMEL3, UKMEL4, and TU1867). The low representation of $C$. meleagridis genomes is due to the limited number of sequencing projects for this species, so all available genomes in public databases until September 2019 were included. The genomes belong to different gp60 subtypes, and the majority comes from the UK. All the studied genomes come from parasite oocysts isolated from human feces of patients with natural infections, and four of these were maintained through passages in piglets, mice, or chickens. Most genomes were sequenced on Illumina's HiSeq and MiSeq platforms, and only two (C. hominis SWEH2 and SWEH5) used the Ion Torrent platform (Supplementary Table 1).

\section{De novo genome assembly analysis}

The raw read data of the 23 selected genomic projects were downloaded from the Sequence Read Archive - SRA of the NCBI public database and assembled with SPAdes. If the isolate had assembly data available, the metrics of the two assemblies were compared, and the contig set that showed the better N50 value was kept for further analysis. Only six genomes of the studied had better N50 values than our assemblies. All of them were deposited at the CryptoDB database v43: C. hominis: UKH1, TU502_2012, 30976, 37999, UdeA01, and C. meleagridis UKMEL1(Supplementary Table 2). Only for three genomes, it was not possible to find a previous assembly data available: $C$. hominis (SWEH2 - SWEH5) and C. meleagridis (TU1867). To exclude possible contaminating contigs, BLASTN comparisons with a Cryptosporidium genome database (reference genomes of $C$. hominis UdeA01, C. parvum Iowa II and C. tyzzeri UGA55) were performed, and only those with Bit score value $\geq 300$ were kept for further analyses. Selected genomes assemblages' statistics are also shown in supplementary table 2. Cryptosporidium genome size is close to $9.0 \mathrm{Mb}$ in most studied isolates; only three have genome assemblies below $9.0 \mathrm{Mb} ; 8.2 \mathrm{Mb}$, in C. parvum anthroponotic UKP14, and $8.8 \mathrm{Mb}$ in both $C$. hominis SWEH2 and SWEH5 isolates. Four genomes had a mean read coverage below 
230

231

232

233

234

235

236

237

238

239

240

241

242

243

244

245

246

247

248

249

250

251

252

253

254

255

256

257

258

259

260

261

262

263

264

265

266

267

268

269

270

271

272

273

274

275

50X, three of which belong to $C$. hominis species (UKH3, SWEH2, and SWEH5) and the $C$. parvum isolate UKP5. The most fragmented genomes were in the $C$. parvum species, isolate UKP3 and the anthroponotic UKP14 with 2,971 and 2,787 contigs, respectively, with coverage above $229 \mathrm{X}$ and $69 \mathrm{X}$. All genomes have an average GC content of $30 \%$ and ambiguities that do not exceed 0.26\%. (Supplementary Table 2)

\section{Single Nucleotide Variants detection}

To identify single nucleotide variants -SNVs- throughout the genomes, inter and intra-species comparisons were made aligning the de novo assembled contigs of each isolate against the reference genome of $C$. parvum Iowa II. Cryptosporidium parvum isolates aligned more than $99.2 \%$ of the contig bases to the Iowa reference, except for the anthroponotic genomes, UKP14 and UKP15, that aligned $97 \%$. C. hominis genome data behave similarly, with an aligned ratio that ranges between 99 to $99.38 \%$, except for UKH4 that reached $98 \%$. In C. meleagridis, a lower alignment rate was achieved with an overall rate close to 97\% (Supplementary Table 3). The global nucleotide identity showed the expected results based on the phylogenetic relationship among the three species. While C. parvum genome identity ranged between $99.51 \%$ and $99.93 \%$ within the species, C. hominis genomes showed an identity of around $96.8 \%$ compared to the Iowa reference genome. Cryptosporidium meleagridis confirmed its higher distance with C. parvum with a global identity of around $91.5 \%$.

Single nucleotide variants within the $C$. parvum genomes ranged between 1,595 and 5,752, except for the anthroponotic isolates that showed more than 18,000 SNVs. When C. hominis genomes were compared with the $C$. parvum Iowa II reference, around 220,000 SNVs were detected in each isolate, while in $C$. meleagridis genomes, the number when up to more than 600,000 (Figure 1).

\section{Phylogenomic analysis}

To verify the topology and taxonomic location described in the genus Cryptosporidium, the SNV data was used to generate a nucleotide matrix. Then, an unrooted maximum likelihood (ML) tree was constructed, including the 24 evaluated genomes ( 23 de novo assembled genomes and the reference genome Iowa II). The transversion model (TVM) was selected as the best model of evolution (Supplementary Table 4). The complete matrix comprised 800,861 sites and the best tree had had a likelihood value of -3401691.458 . The tree obtained shows three monophyletic clades, following the actual classification scheme, that group the 24 genomes of the three Cryptosporidium species included in this work. The branch supports are optimal and have a $100 \%$ agreement between SH-aLRT and ultra-fast bootstrap for the main branches, with discrepancies in three internal C. parvum nodes and one internal C. hominis node (Figure 2). Within the $C$. parvum species, the presence of two separate branches with statistical supports was observed, which allowed the segregation of the anthroponotic isolates UKP14 and UKP15 in a separate branch of the zoonotic isolates with a $100 \%$ support. Furthermore, it is noteworthy to point out that all the $C$. parvum isolates that belong to the gp60 gene IIa subtype family grouped with a $100 \%$ bootstrap, including the isolate Iowa II. In the case of $C$. hominis clade, the isolates seem to segregate with statistical support according to its subtype family, except for isolate 30976. The $C$. meleagridis clade is also supported with $100 \%$ bootstrap, and the phylogenetic signal is enough to separate the 4 different isolates according to its subtype family. In general, all genomes were grouped according to the species classification and its gp60 gene subtype family (Figure 2). 


\section{Single Nucleotide Variants in coding regions}

278 SNVs located in coding regions were identified in all the genomes through the read mapping

279 analysis against the $C$. parvum Iowa II version 43 of CryptoDB. As expected, compared with the

280

281

282

283

284

285

286

287

288

289

290

291

292

293

294

295

296

297

298

299

300

301

302

303

304

305

306

307

308

309

310

311

312

313

314

315

316

317

318

319

320

321 C. parvum IOWA reference, the genomes of $C$. hominis and $C$. meleagridis showed the highest number of these variants in coding regions with more than 150,000 and 400,000, respectively, and nearly $60 \%$ corresponding to synonymous changes (sSNVs). There were no intraspecies differences in the accumulation of variants in coding regions or synonymous and nonsynonymous changes in both species. On the contrary, in C. parvum isolates, the number of SNVs in CDSs was less than 20,000 in all genomes, with more than 50\% corresponding to nonsynonymous mutations. The results in C. parvum suggest an intraspecies heterogeneity with values ranging between 840 and 3,476 SNVs in CDSs in zoonotic isolates and around 14,000 in anthroponotic isolates. However, these differences were not statistically significant. After characterizing the synonymous and non-synonymous mutations in coding regions, the genes with nsSNVs were analyzed separately, finding 2,532 in at least one genome of each species, with 110 exclusives for C. meleagridis, 11 for C. hominis, and 2 for C. parvum.

Genes with non-synonymous variants (nsSNVs) were annotated by the SIFT4G effect predictor, and those with non-tolerated changes and possibly associated with deleterious mutations were identified. With this strategy, a total of 1,017 genes were predicted with deleterious mutations in C. meleagridis, 715 in C. hominis, and 288 in C. parvum. Of these, 183 are shared by all three species, being present in at least one genome of each species; 377 are exclusive to $C$. meleagridis; 103 to C. hominis, and 33 to C. parvum (Figure 3). Only $29.5 \%$ of the genes annotated with deleterious mutations by SIFT, and shared by the three species, could be annotated with EggNOG mapper. Enrichment analysis in GO terms indicated that they are mainly involved in biological processes and metabolic processes with molecular functions such as catalytic or binding activity.

The functional orthology analysis performed on the KAAS server against the KEGG database had a better performance compared to the previous GO assignment, annotating $46.5 \%$ of the shared genes and indicating that most of them are involved in enzymatic processes, DNA repair, recombination, and proteins associated with the chromosomes (Figure 4). Complementary annotation determined that only $1.1 \%$ of the genes encoded proteins secreted by the classical pathway, whereas $10.9 \%$ encoded for proteins secreted by non-classical systems. Additionally, $2.3 \%$ of the putative encoded proteins exhibited domains with transmembrane helixes.

Regarding genes that carry deleterious mutations within each species (species-specific mutated genes), 29.1 and $28.9 \%$ were classified in at least one GO category for $C$. hominis and $C$. meleagridis, respectively. In C. parvum, GO terms were assigned only in 3 genes $(9.09 \%)$, classified exclusively in the cellular component category. The functional orthology analysis again achieved a better result compared to the EggNOG-mapper software, assigning $42.4 \%$ in $C$. parvum (Figure 5, panel A), 60.2\% KO in C. hominis (Figure 5, panel B), and 59.1\% in $C$. meleagridis (Figure 5, panel C). The assignment of functional orthologs in metabolic pathways established that in the three species, most of these genes are involved in enzymatic processes. The second most frequently assigned pathway in genes with species-exclusive deleterious mutations was ribosome transfer RNA biogenesis in C. parvum, spliceosome complex in $C$. hominis, and biogenesis in C. meleagridis.

\section{Indel events analysis}


322

323

324

325

326

327

328

329

330

331

332

333

334

335

336

337

338

339

340

341

342

343

344

345

346

347

348

349

350

351

352

353

Inter and intraspecies indel events were identified in the genomes through their comparison to the C. parvum Iowa II reference genome. For simplicity reasons, insertions and deletions were referred, assuming as reference the C. parvum Iowa II genome. Observed insertions were in the range of 50 to 500 nucleotides. In C. parvum, the highest number of insertions was detected in the zoonotic isolates UKP3 and UKP4. No statistically significant differences were found in the accumulation of insertions among the studied species. Deletions identified in the genomes had a similar size to that described for the insertions, with the majority falling into the range of 50 to 500 nucleotides. The highest number was observed in the genomes of $C$. meleagridis, followed by $C$. hominis. However, no significant differences were found in the accumulation of deletions among species.

Coding genes that presented indels in the three species were identified and annotated, whenever possible. We found 322, 215, and 176 genes with indels in C. hominis, C. parvum, and C. meleagridis, respectively, but only 13 were common to all 23 Cryptosporidium isolates. In both C. meleagridis and C. hominis, deletions were the most frequent indel event, corresponding to $76.1 \%$ and $59 \%$ of all structural variants, respectively. The C. hominis isolates UKH4 was the exception, in which insertions predominated. In contrast, in C. parvum, deletions were less common, representing $38 \%$ of the structural variants detected in the CDSs.

To gain insights into the gene losses occurring in the 23 isolates, those genes with indel events present in at least two genomes/isolates in each species were characterized. A total of 116 genes were selected in C. meleagridis, 100 in C. parvum, and 66 in C. hominis. Sixty-three were exclusive to C. meleagridis, 62 to C. parvum, and 14 to C. hominis (Figure 6).

According to the annotation deposited in CryptoDB Version 46, of the 13 genes with insertion and deletion events shared by the three species, $40 \%$ code for uncharacterized proteins and the remaining for hydrolases (cgd8_1220), proteins with recognition motifs of RNA (cgd3_4150), mucins (cgd7_4020), among others. Twenty-seven genes with indels were identified in all $C$. meleagridis genomes, with $56 \%$ of them corresponding to uncharacterized proteins. Regarding C. parvum, insertions in the genes cgd3_190 (involved in the formation of fibrillin) and cgd3_3900 (uncharacterized protein) were present in $56 \%$ of the evaluated genomes. An interesting finding was that all $C$. hominis genomes exhibited indels in three genes (orthologs of cgd6_4290, cgd7_420, and cgd7_500); with cgd6_4290 and cgd7_420 being also affected in all genomes of C. meleagridis (Supplementary Table 5). 


\section{Discussion}

355 Advances in DNA sequencing technologies and bioinformatics have promoted the routine use of

356 complete genome sequences, revolutionizing the study of both model and non-model organisms,

357 particularly in microbiology (Young \& Gillung, 2020). Phylogenomic is one of the numerous

358 disciplines that have taken advantage of the progress in NGS technologies, using the massive

359 datasets to infer both phylogenetic relationships between taxa, improving the understanding of

360 molecular evolution, and putative functions for DNA or protein sequences (Young \& Gillung,

361 2020).

362 A phylogenomic analysis is being applied now to validate previous findings with classic singlemarker molecular methods and to resolve with unprecedented resolution, the phylogenetic relationships between species and isolates of the human infecting Cryptosporidium parasites (Glaberman et al., 2001; Abe \& Makino, 2010; Abal-Fabeiro et al., 2013; Wagnerová et al., 2015; Pérez-Cordón et al., 2016). However, this phylogenomic approach has focused on C. hominis and C. parvum by studying multiple concatenated loci (Feng et al., 2017; Nader et al., 2019), or different variable positions that represent below 1\% of the genome (Gilchrist et al., 2018). To gain insights into the evolutionary relationships in Cryptosporidium, a comparative and phylogenomic study was conducted with 23 genomes of the most frequent species in humans: $C$. hominis, $C$. parvum, and C. meleagridis.

372 The sequence identity between $C$. hominis genomes $(96.85 \%)$ and the reference genome of $C$. parvum Iowa II found in our study confirms the high similarity between both species and agrees with previous findings that report differences of a maximum of 3\% (Xu et al., 2004; Mazurie et al., 2013b; Zahedi et al., 2013). These studies describe that both genomes exhibit remarkable structural conservation, and some authors suggest that the phenotypic differences may be due to subtle variations in the sequences of genes that code for the interface proteins between the parasite and its host (Xu et al., 2004; Mazurie et al., 2013b; Zahedi et al., 2013). Regarding $C$. parvum, there was a lower percentage of aligned blocks against the reference genome in the anthroponotic isolates compared to the zoonotic ones, which agrees with other studies that have also described differences in the genomic structure and nucleotide content between zoonotic and anthroponotic isolates of C. parvum (Widmer et al., 2012; Fan, Feng \& Xiao, 2019; Nader et al., 2019). As it was expected, $C$. meleagridis genomes had a lower global identity against $C$. parvum reference genome than that detected with $C$. hominis, which agrees with the phylogenetic relationships described for the three species using different genetic locus (Šlapeta, 2013; Khan, Shaik \& Grigg, 2017). This finding may be related to the differences in host specificity reported in the three species, where $C$. hominis and $C$. parvum have host ranges limited to mammals, while C. meleagridis is described in mammals and birds (Šlapeta, 2013; Khan, Shaik \& Grigg, 2017). It has been suggested that mammals were possibly the original hosts for $C$. meleagridis and that later this species adapted to birds (Caccio \& Widmer, 2014). Single nucleotide variants (SNVs) results obtained in this study showed similar behavior in their number and frequency in the genomes of $C$. hominis with around 220,000 variants, compared to the $C$. parvum IOWA reference, results that differ from those reported in previous comparative studies (Isaza et al., 2015; Gilchrist et al., 2018). Isaza et al. (Isaza et al., 2015) identified an average of 43,258 SNVs between the reference genome $C$. parvum Iowa II and the $C$. hominis UdeA01, UKH1, and TU502_2012 isolates (deposited in CryptoDB version 8). One possible explanation of the lower number of SNVs detected in this work could be the different methodological approaches used to identify these variants. Authors only considered those SNVs 
400 total number of SNVs. Additionally, the genomes used in Isaza's study were obtained from the

401 CryptoDB version 8 database, whose genomes had a lower degree of purification than those used

402 in our work (version 43). Gilchrist et al. (Gilchrist et al., 2018) also found a lower number of

403 SNVs $(36,780)$ in a comparative study of 32 genomes of $C$. hominis. The authors mapped the

404 short reads of the $C$. hominis genomes against the genome of $C$. parvum Iowa, which was

405

406

407

408

409

410 sequenced with Pacific Biosciences (PacBio). It could be possible that the assembly of a genome through long sequencing reads can affect the number of variants detected. Although it is not clear how long reads sequencing methods might differ from comparative genomic approaches using short-read data, i.e., Illumina (De Maio et al., 2019), it has been shown that error rates on this platform are much higher than those recorded for Illumina (15\% versus $0.1 \%$ ) (Watson, 2018) and usually is performed at a lower read coverage compare to Illumina projects.

411 Concerning the single nucleotide variants among the $C$. parvum genomes evaluated in this study, 412 a more significant number of SNVs was identified in the anthroponotic isolates with more than 41318,000 compared to the zoonotic isolates, which ranged between 1,595 and 5,752 SNVs. These results are similar to the findings reported by Widmer et al. (Widmer et al., 2012), who identified about 16,606 SNVs by comparing the genomes of two $C$. parvum isolates with different host ranges, the anthroponotic $C$. parvum TU114 and the reference genome of the zoonotic Iowa II isolate. The differential accumulation of intragenotypic SNVs in C. parvum reflects the genetic diversity of this species, which is possibly related to the evolution of the parasite influenced by the selective pressures in both humans and animal hosts. For this reason, it has been postulated that the accumulation of genomic variants could influence the host range (Weir et al., 2011; Blake et al., 2015; Grinberg \& Widmer, 2016). Concerning C. meleagridis, more than 600,000 SNVs were identified in the genomes compared against the reference genome of $C$. parvum. It is essential to highlight that there are currently no reports on the structural variations like SNVs in this species, being this the first one.

425 In this work, a comprehensive phylogenomic analysis using more than 800.000 single nucleotide variations detected in 24 genomes of the species $C$. parvum, C. hominis, and C. meleagridis was done. To our knowledge, this is the most extensive phylogenomic analysis carried out within the genus and one of the largest within the Phylum Apicomplexa. One of the main findings in this study is that, although we didn't select neutral evolving positions within the genomes, there was a strong phylogenetic signal, supported by two statistical tests, that allowed the well-supported segregation for most of the isolates. However, discrepancies were found in three internal $C$. parvum nodes and one in $C$. hominis. It is essential to highlight that the mentioned differences did not alter the global topology of the phylogenomic tree obtained. In related literature, it has been described that the mismatches in the supports of the branches obtained by bootstrapping and probability ratio tests can arise as a consequence of performing the analysis with small samples and with highly heterogeneous nucleotide substitution models (Guindon et al., 2010). The obtained tree confirms the previously reported topology for these three intestinal species of Cryptosporidium, inferred from single-locus phylogenetic studies, and by the use of different loci such as 18S rRNA, gp60, and other polymorphic genes (Ren et al., 2012; Šlapeta, 2013; Nader et al., 2019). Cryptosporidium meleagridis is confirmed as the most divergent group among the three studied species (Šlapeta, 2013; Khan, Shaik \& Grigg, 2017). At the species rank, the bootstrap and SH-aLRT statistical support was $100 \%$. Furthermore, most of the isolates were grouped according to its gp60 gene family type, regardless of its geographical origin. The phylogenomic analysis of the $C$. parvum isolates evidenced the separation of its central clade into two branches with significant statistical support (100\%), with zoonotic isolates in one 
446 branch and anthroponotic isolates in the other. This finding agrees with that reported by several 447 authors (Widmer et al., 2012; Danišová et al., 2017; Feng et al., 2017; Nader et al., 2019), in 448 which through unilocus phylogenetic analyzes and multiple concatenated loci between 449 anthroponotic and zoonotic isolates, determined that the anthroponotic isolates of C. parvum 450 formed a separate group from the zoonotic isolates. Nader et al. (Nader et al., 2019), through 451 phylogenetic analysis of neutrally evolving coding loci across 21 Cryptosporidium isolates, 452 identified two $C$. parvum lineages with distinct host-specificity, which were designated as 453 Cryptosporidium parvum parvum (zoonotic) and C. p. anthroponosum (anthroponotic).

454 Additionally, they found that human infective C. hominis and C. parvum isolates form a distinct superclade along with $C$. cuniculus, another species associated with human infections.

Subsequent analysis of high-quality SNPs detected in 16 genomes of the two Cryptosporidium parvum subclades, confirming the zoonotic C. p. parvum and anthroponotic C. p. anthroponosum subspecies designation (Nader et al., 2019).

459 Feng et al. (Feng et al., 2017) evaluated the SNVs accumulation in several genomes of $C$. 460 parvum IIa and IId families, which preferentially infect calves and lambs, respectively, in some 461 European countries. This study revealed that most of the SNVs occur in subtelomeric regions of 462 the chromosomes, with a high percentage located in coding regions of the genome, and near the 463 half being non-synonymous. Additionally, the subtypes evaluated shared more than $50 \%$ of 464 SNVs, and phylogenetic analysis of the SNVs data showed a robust separation of IIa sequences 465 and IId sequences, and a high divergence with reference Iowa genome (Feng et al., 2017). These findings agree with the results obtained in this study because SNVs show a concordant relationship with gp60 subfamily typing. Conversely, Gilchrist et al. (Gilchrist et al., 2018) studied the genetic diversity of thirty-two genomes of different $C$. hominis subtypes isolated from children with poor living conditions from Bangladesh. They found 36,780 SNVs that varied between the $C$. hominis isolates, with a homogeneous distribution throughout the genome and only $4 \%$ occurring with a frequency greater than 20\%. These authors also built a phylogenetic tree based on the SNVs $(1,582)$ found in those genomes, in which no groupings regarding the subtype family was observed, concluding that the use of a single marker (gp60) does not reflect the evolutionary changes of the entire genome and, in turn, confirming the weakness of the typing of unique markers for taxonomic assignments within this genus. Our findings reinforce this argument since an analysis with a more significant number of positions of different isolates considerably improves the resolution power compared to that obtained from unilocus analysis or multiple concatenated loci or partial fragments of the genome. Another aspect that could influence the topology of the phylogenomic tree obtained by Gilchrist et al. is the high rate of recombination on chromosome 6 reported among circulating isolates from endemic countries for $C$. hominis (Li et al., 2013; Zahedi et al., 2013). This feature is associated with greater genetic variability and the generation of hypertransmittable subtypes and favors a wide distribution of gp60-based allelic families in the phylogenetic tree without a cluster aggregation. The recombination phenomenon was not a relevant variable in our study since genomes analyzed correspond to isolates circulating in different geographic locations of four continents.

487 Several authors have proposed that phenotypic differences between Cryptosporidium species are 488 related to polymorphisms on protein-coding regions (Xu et al., 2004; Pain, Crossman \& Parkhill, 489 2005; Bouzid et al., 2013; Nader et al., 2019). In our study, the single nucleotide variants located 490 in CDSs were analyzed and characterized as synonymous and non-synonymous changes.

491 Compared to the C. parvum reference, Cryptosporidium species that showed the highest number 
492 of SNVs in protein-coding regions were $C$. meleagridis and $C$. hominis than 400,000 and 493150,000 variants, respectively. Forty-two percent of them corresponded to non-synonymous 494 changes. Our results on the number of SNVs in C. hominis differs from the data reported previously, and similarly, the same occurs with SNVs in coding regions. Isaza et al. (Isaza et al., 2015) identified 36,753 SNVs located on coding regions in the genome of four isolates of $C$. hominis (version 8 of CryptoDB), using as a reference the genome of C. parvum Iowa II. As we mentioned before, these discrepancies may be related to the methodological approach used in every study.

In C. parvum, the number of variants in coding regions was below 20,000 SNVs, with intraspecies differences related to a heterogeneous behavior between zoonotic and anthroponotic isolates. Additionally, it was identified that more than $50 \%$ of the SNVs located in coding regions correspond to non-synonymous mutations. In a previous study carried out by Widmer and Col. (Widmer et al., 2012), non-synonymous SNVs were present in a range from $28 \%$ to 32 $\%$ of all SNVs, and $60 \%$ of all nucleotide positions in the two genomes were not synonymous. Analysis of the deleterious mutations in the Cryptosporidium species evaluated in the study showed that 183 genes were predicted with mutations that were present in at least one genome of each species: 377 exclusive to C. meleagridis, 103 to C. hominis, and 33 to C. parvum. Unfortunately, $53,5 \%$ of the genes with deleterious changes were located in uncharacterized coding regions (hypothetical proteins), so the biological impact of these mutations could not be determined. Annotation of the shared and species-exclusive genes with deleterious mutations establish that most encode enzymes and proteins involved in DNA repair, recombination processes, proteins associated with the chromosomes, as well as the biogenesis of transfer RNA and ribosomes. Comparative analyzes carried out previously have found that the genes with the highest number of SNVs in C. hominis and C. parvum were related to ribosome assembly, translation processes, and coding genes for proteins with transmembrane domains (Widmer et al., 2012; Isaza et al., 2015). Sikora et al. (Sikora et al., 2017b) carried out an intraspecies comparative analysis of 14 genomes of $C$. hominis. They found 18 genes with non-synonymous mutations, with only the gene that codes for a protein of the oocyst wall COWP9 (oocyst of cgd6_210) with a deleterious mutation, which was present in eight of the fourteen genomes. Mutated genes annotated in our study also code for surface proteins with transmembrane domains and proteins secreted by non-classical pathways, suggesting that the interaction processes between the parasite and the host cells could be affected. This finding agrees with that reported by other authors who have described that the processes that are mainly affected are adhesion and invasion (Li et al., 2013; Isaza et al., 2015; Gilchrist et al., 2018; Widmer, 2018; $\mathrm{Xu}$ et al., 2019). It has been determined that the proteins secreted by non-classical pathways usually are growth factors, inflammatory cytokines, components of the extracellular matrix that regulate cell differentiation, proliferation, and apoptosis, as well as surface proteins in parasites involved in the initial interaction with the host (Nickel, 2003). This reinforces the need to improve the annotation of Cryptosporidium genomes, allowing the understanding of unknown aspects related to evolution, virulence, and pathogenicity in this genus.

Indels events in the genomes of the 23 Cryptosporidium isolates were also evaluated in our study. More than $60 \%$ of these variants were located in CDSs, a finding that could be expected since the Cryptosporidium genome has a percentage of coding regions more significant than $70 \%$ (Nader et al., 2019; Xu et al., 2019). The highest number of deletions, using reference C. parvum IOWA, occurred in C. meleagridis genomes, followed by $C$. hominis, suggesting a partial loss of genome fragments these species. Indel events were less abundant than SNVs in the 23 genomes 
538

539

540

541

542

543

544

545

546

547

548

549

550

551

552

553

554

555

556

557

558

559

560

561

562

563

564

565

566

567

568

569

570

571

572

573

574

575

576

577

578

579

580

581

582

583

analyzed, contrary to the reports in other apicomplexan such as Plasmodium falciparum, in which they have been described as the dominant mechanism of polymorphism within the genome (Miles et al., 2016). Feng et al. (Feng et al., 2017) identified 1,200 insertion events and 1,500 deletions in a comparative genomic study of $C$. parvum isolates. In our study, which analyzed a more extensive number of genomes, we found less than 100 structural variations in each of the zoonotic and anthroponotic isolates. Previous studies have determined that indels are significantly more frequent in the peri-telomeric and subtelomeric regions of Cryptosporidium genomes (Nader et al., 2019). Guo et al. (Guo et al., 2015) analyzed five genomes of C. hominis against the reference $C$. parvum Iowa, and they identified several insertions and deletions near the telomeres on chromosome 6 , associated with recombination events, which could indicate that the duplication or deletion of subtelomeric genes is involved in the differences in host specificity between Cryptosporidium species. These recombination events can also explain the low support obtained in the phylogeny for the $C$. hominis 30976 isolate. Members of multicopy gene families and under a strong positive selection, such as MEDLE proteins, insulin-like proteases, and mucin-type glycoproteins, related to the parasite-host interaction, are ubicated in these regions (Fan, Feng \& Xiao, 2019; Feng \& Xiao, 2019; Xu et al., 2019).

Although several studies have described deletions in genes encoding the MEDLE proteins in $C$. parvum and C. hominis (Widmer et al., 2012; Liu et al., 2016), in the present study, no structural variants were found in these genes or those encoding insulin-like proteases. However, deletions were found in all genomes of $C$. meleagridis and at least two isolates of $C$. hominis and $C$. parvum in a gene coding for a cryptosporidial mucin (ortholog of cgd7_4020), also known as gp900. This is a microneme secreted surface glycoprotein encoded by a single copy gene; it is involved in the apical portion of sporozoites and merozoites to enterocytes, which is required to initiate the invasion process (Okhuysen \& Chappell, 2002; Carruthers \& Tomley, 2008). However, since there is a repertoire of adhesion proteins in Cryptosporidium, including the proteins related to thrombospondin, p23, the gp40 / p30 protein complex, and the Circumsporozoite-like protein - CSL (Langer-Curry \& Riggs, 1999; Bouzid et al., 2013), the alterations identified in the gp900 gene probably do not affect the interaction processes between the invasive stages of the parasite and the host cell. Additional analyzes are required to determine the biological implications of these deletions in the binding and invasion process, mainly in isolates of $C$. meleagridis.

Another interesting finding in this study was identifying deletions in all genomes of $C$. meleagridis and C. hominis for genes that encode for proteins with a WD-40 (cgd6_4290 ortholog) and SNF2/DEXDc/HELICc domains (cdg7_420 ortholog). WD-40 domain has tryptophan-aspartic acid (WD) repeats of approximately 40 amino acids and is considered one of the ten most abundant protein domains in eukaryotes (Xu \& Min, 2011; Jain \& Pandey, 2018). The proteins that contain these repeats are involved in various cellular processes, acting as an adaptor in many different protein complexes or protein-DNA complexes, signal transduction, transcription, cell cycle regulation, and apoptosis; however, no enzymatic activity has been assigned (Stirnimann et al., 2010; Xu \& Min, 2011). These domains have been reported as highly polymorphic in other apicomplexan protozoa, such as Plasmodium falciparum, suggesting the participation of WD-40 in basic cellular and metabolic processes (Chahar et al., 2015). SWI2/SNF2 (Switching defective -SWI and Sucrose nonfermenting-SNF) protein family are ATP-dependent chromatin remodeling factors that modulate the access of transcription factors to regulatory regions of genes (Sullivan et al., 2013). Previous reports indicate homologs of these domains in different apicomplexan, including Plasmodium falciparum (Ji \& Arnot, 1997) and

Peer] reviewing PDF | (2020:05:49002:1:1:NEW 8 Oct 2020) 
584 Toxoplasma gondii (Sullivan et al., 2003). Cryptosporidium parvum has 14 chromatin-

585 remodeling SNF2/SWI2 ATPases (Templeton et al., 2004). Alterations in the genes encoding

586 these proteins could affect the epigenetic regulatory mechanisms in this genus.

587 Exclusive insertions were identified in 50\% of the C. parvum isolates in the cgd3_190 gene. This

588 gene encodes a microneme secreted protein with epidermal growth factor - EGF domains like,

589 which are involved in cell signaling (Carruthers \& Tomley, 2008). In other apicomplexans, such

590 as Toxoplasma gondii, these domains have been associated with adhesion processes to the host

591 cell (Huynh, Boulanger \& Carruthers, 2014). It has also been shown that in the presence of

592 calcium, the EGF domains adopt an extended structure resistant to proteases, favoring the

593 interaction of the N-terminal portion of the molecule with the host cell ligands, favoring invasion

594 (Carruthers \& Tomley, 2008).

595

596

597

598

599

600

601

602

603

604

605

606

607

608

609

610

611

612

613

614

615

616

617

618

619

620

621

622

623

624

625

626

627

628

629

630

631

\section{Conclusions}

Here we present, to our knowledge, the most comprehensive phylogenomic and genomic comparative analysis performed in the most relevant human infecting Cryptosporidium species, which includes complete genomes from different isolates, allelic families, and subtypes. Comparative analysis of more than 800,000 single nucleotide variable positions detected in 24 genomes of the three main species infecting humans (C. parvum, C. hominis, and C. meleagridis) generate a more robust analysis on the phylogenetic relationships between the Cryptosporidium species of human public health concern. This phylogenomic analysis also confirmed the gp60 loci segregation pattern observed in subtype families. Most of the SNVs and indels detected in the study genomes were ubicated in coding regions. Genes with deleterious changes and indels were identified and annotated, whenever possible, in the three species. These mutated genes were associated with the processing of genetic information and enzymatic and metabolic processes; however, most of them remain uncharacterized and encode hypothetical proteins.

\section{Funding}

This work was funded by the COLCIENCIAS Convocatoria 777-2017 Para Proyectos de Ciencia, Tecnología e Innovación en Salud 2017, code 115-777 57608, and Vicerrectoría de Investigación (CODI), Universidad de Antioquia, code 2017-16-171.

\section{References}

Abal-Fabeiro JL, Maside X, Bello X, Llovo J, Bartolomé C. 2013. Multilocus patterns of genetic variation across Cryptosporidium species suggest balancing selection at the gp60 locus. Molecular Ecology 22:4723-4732. DOI: 10.1111/mec.12425.

Abe N, Makino I. 2010. Multilocus genotypic analysis of Cryptosporidium isolates from cockatiels, Japan. Parasitology Research 106:1491-1497. DOI: 10.1007/s00436-010-1810-5.

Abrahamsen MS. 2004. Complete Genome Sequence of the Apicomplexan, Cryptosporidium parvum. Science 304:441-445. DOI: 10.1126/science.1094786.

Adamu H, Petros B, Zhang G, Kassa H, Amer S, Ye J, Feng Y, Xiao L. 2014. Distribution and Clinical Manifestations of Cryptosporidium Species and Subtypes in HIV/AIDS Patients in Ethiopia. PLoS Neglected Tropical Diseases 8. DOI: 10.1371/journal.pntd.0002831.

Almagro Armenteros JJ, Tsirigos KD, Sønderby CK, Petersen TN, Winther O, Brunak S, von Heijne G, Nielsen H. 2019. SignalP 5.0 improves signal peptide predictions using deep neural networks. Nature Biotechnology 37:420-423. DOI: 10.1038/s41587-019-0036-z.

Andrew Rambaut. 2018. FigTree, version 1.4.4. Computer program distributed by the author, website: http://tree.bio.ed.ac.uk/software/figtree/[accessed enero, 2020].

Baele G, Suchard MA, Rambaut A, Lemey P. 2017. Emerging concepts of data integration in pathogen

Peer) reviewing PDF | (2020:05:49002:1:1:NEW 8 Oct 2020) 
632

633

634

635

636

637

638

639

640

641

642

643

644

645

646

647

648

649

650

651

652

653

654

655

656

657

658

659

660

661

662

663

664

665

666

667

668

669

670

671

672

673

674

675

676

677

678

679

680

681

682

phylodynamics. In: Systematic Biology. e47-e65. DOI: 10.1093/sysbio/syw054.

Bankevich A, Nurk S, Antipov D, Gurevich AA, Dvorkin M, Kulikov AS, Lesin VM, Nikolenko SI, Pham SON, Prjibelski AD, Pyshkin A V, Sirotkin A V, Vyahhi N, Tesler G, Alekseyev MAXA, Pevzner PA. 2012. SPAdes: A New Genome Assembly Algorithm and Its Applications to SingleCell Sequencing. 19:455-477. DOI: 10.1089/cmb.2012.0021.

Bendtsen JD, Jensen LJ, Blom N, Von Heijne G, Brunak S. 2004. Feature-based prediction of nonclassical and leaderless protein secretion. Protein Engineering, Design and Selection 17:349-356. DOI: $10.1093 /$ protein/gzh037.

Beser J, Hallström BM, Advani A, Andersson S, Östlund G, Winiecka-Krusnell J, Lebbad M, Alm E, Troell K, Arrighi RBG. 2017. Improving the genotyping resolution of Cryptosporidium hominis subtype IbA10G2 using one step PCR-based amplicon sequencing. Infection, Genetics and Evolution 55:297-304. DOI: 10.1016/j.meegid.2017.08.035.

Blake DP, Clark EL, Macdonald SE, Thenmozhi V, Kundu K, Garg R, Jatau ID, Ayoade S, Kawahara F, Moftah A, Reid AJ, Adebambo AO, Zapata RÁ, Rao ASRS, Thangaraj K, Banerjee PS, DhinakarRaj G, Raman M, Tomley FM. 2015. Population, genetic, and antigenic diversity of the apicomplexan Eimeria tenella and their relevance to vaccine development. Proceedings of the National Academy of Sciences of the United States of America 112:E5343-E5350. DOI: 10.1073/pnas.1506468112.

Bouzid M, Hunter PR, Chalmers RM, Tyler KM. 2013. Cryptosporidium pathogenicity and virulence. Clinical Microbiology Reviews 26:115-134. DOI: 10.1128/CMR.00076-12.

Caccio SM, Widmer G. 2014. Cryptosporidium: parasite and disease.

Cama VA, Bern C, Roberts J, Cabrera L, Sterling CR, Ortega Y, Gilman RH, Xiao L. 2008. Cryptosporidium species and subtypes and clinical manifestations in children, Peru. Emerging Infectious Diseases 14:1567-1574. DOI: 10.3201/eid1410.071273.

Cama VA a, Ross JMM, Crawford S, Kawai V, Chavez-Valdez R, Vargas D, Vivar A, Ticona E, Navincopa M, Williamson J, Ortega Y, Gilman RHH, Bern C, Xiao L, Chavez-Valdez R, Vargas D, Vivar A, Ticona E, Navincopa M, Williamson J, Ortega Y, Gilman RHH, Bern C, Xiao L. 2007. Differences in Clinical Manifestations among Cryptosporidium Species and Subtypes in HIV-Infected Persons . The Journal of Infectious Diseases 196:684-691. DOI: 10.1086/519842.

Carruthers VB, Tomley FM. 2008. Microneme proteins in apicomplexans. Sub-Cellular Biochemistry 47:33-45. DOI: 10.1007/978-0-387-78267-6_2.

Chahar P, Kaushik M, Gill SS, Gakhar SK, Gopalan N, Datt M, Sharma A, Gill R. 2015. Genome-wide collation of the Plasmodium falciparum WDR protein superfamily reveals malarial parasite-specific features. PLoS ONE 10:1-24. DOI: 10.1371/journal.pone.0128507.

Cornillot E, Hadj-Kaddour K, Dassouli A, Noel B, Ranwez V, Vacherie B, Augagneur Y, Brès V, Duclos A, Randazzo S, Carcy B, Debierre-Grockiego F, Delbecq S, Moubri-Ménage K, Shams-Eldin H, Usmani-Brown S, Bringaud F, Wincker P, Vivarès CP, Schwarz RT, Schetters TP, Krause PJ, Gorenflot A, Berry V, Barbe V, Mamoun C Ben. 2012. Sequencing of the smallest Apicomplexan genome from the human pathogen Babesia microti. Nucleic Acids Research 40:9102-9114. DOI: 10.1093/nar/gks700.

Cunha FS, Peralta JM, Peralta RHS. 2019. New insights into the detection and molecular characterization of Cryptosporidium with emphasis in Brazilian studies: A review. Revista do Instituto de Medicina Tropical de Sao Paulo 61. DOI: 10.1590/s1678-9946201961028.

Danecek P, Auton A, Abecasis G, Albers CA, Banks E, DePristo MA, Handsaker RE, Lunter G, Marth GT, Sherry ST, McVean G, Durbin R. 2011. The variant call format and VCFtools. Bioinformatics 27:2156-2158. DOI: 10.1093/bioinformatics/btr330.

Danišová O, Valenčáková A, Stanko M, Luptáková L, Hatalová E, Čanády A. 2017. Rodents as a reservoir of infection caused by multiple zoonotic species/genotypes of C. parvum, C. hominis, C. suis, C. scrofarum, and the first evidence of C. muskrat genotypes I and II of rodents in Europe. Acta Tropica 172:29-35. DOI: 10.1016/j.actatropica.2017.04.013.

Delcher AL, Phillippy A, Carlton J, Salzberg SL. 2002. Fast algorithms for large-scale genome alignment

Peer) reviewing PDF | (2020:05:49002:1:1:NEW 8 Oct 2020) 
683

684

685

686

687

688

689

690

691

692

693

694

695

696

697

698

699

700

701

702

703

704

705

706

707

708

709

710

711

712

713

714

715

716

717

718

719

720

721

722

723

724

725

726

727

728

729

730

731

732

733

and comparison. 30:2478-2483.

Efstratiou A, Ongerth JE, Karanis P. 2017. Waterborne transmission of protozoan parasites: Review of worldwide outbreaks - An update 2011-2016. Water Research 114:14-22. DOI: 10.1016/j.watres.2017.01.036.

Fan Y, Feng Y, Xiao L. 2019. Comparative genomics: how has it advanced our knowledge of cryptosporidiosis epidemiology? Parasitology Research 118:3195-3204. DOI: 10.1007/s00436019-06537-x.

Felsenstein J. 1981. Evolutionary trees from DNA sequences: A maximum likelihood approach. Journal of Molecular Evolution 17:368-376. DOI: 10.1007/BF01734359.

Feng Y, Alderisio KA, Yang W, Blancero LA, Kuhne WG, Nadareski CA, Reid M, Xiao L. 2007. Cryptosporidium genotypes in wildlife from a New York watershed. Applied and Environmental Microbiology 73:6475-6483. DOI: 10.1128/AEM.01034-07.

Feng Y, Li N, Roellig DM, Kelley A, Liu G, Amer S, Tang K, Zhang L, Xiao L. 2017. Comparative genomic analysis of the IId subtype family of Cryptosporidium parvum. International Journal for Parasitology 47:281-290. DOI: 10.1016/j.ijpara.2016.12.002.

Feng Y, Ryan UM, Xiao L. 2018. Genetic Diversity and Population Structure of Cryptosporidium. Trends in Parasitology. DOI: 10.1016/j.pt.2018.07.009.

Feng Y, Xiao L. 2019. Differential Expression of Three Cryptosporidium Species-Specific MEDLE Proteins. 10:1-11. DOI: 10.3389/fmicb.2019.01177.

Firoozi Z, Sazmand A, Zahedi A, Astani A, Fattahi-Bafghi A, Kiani-Salmi N, Ebrahimi B, DehghaniTafti A, Ryan U, Akrami-Mohajeri F. 2019. Prevalence and genotyping identification of Cryptosporidium in adult ruminants in central Iran. Parasites and Vectors. DOI: 10.1186/s13071019-3759-2.

Galen SC, Borner J, Martinsen ES, Schaer J, Austin CC, West CJ, Perkins SL. 2018. The polyphyly of Plasmodium: Comprehensive phylogenetic analyses of the malaria parasites (Order Haemosporida) reveal widespread taxonomic conflict. Royal Society Open Science 5. DOI: 10.1098/rsos.171780.

Gilchrist CA, Cotton JA, Burkey C, Arju T, Gilmartin A, Lin Y, Ahmed E, Steiner K, Alam M, Ahmed S, Robinson G, Zaman SU, Kabir M, Sanders M, Chalmers RM, Ahmed T, Ma JZ, Haque R, Faruque ASG, Berriman M, Petri WA. 2018. Genetic diversity of cryptosporidium hominis in a bangladeshi community as revealed by whole-genome sequencing. Journal of Infectious Diseases 218:259-264. DOI: 10.1093/infdis/jiy121.

Glaberman S, Sulaiman IM, Bern C, Limor J, Peng MM, Morgan U, Gilman R, Lal AA, Xiao L. 2001. A multilocus genotypic analysis of Cryptosporidium meleagridis. Journal of Eukaryotic Microbiology Suppl:19S-22S.

Grinberg A, Widmer G. 2016. Cryptosporidium within-host genetic diversity: systematic bibliographical search and narrative overview. International Journal for Parasitology 46:465-471. DOI: 10.1016/j.ijpara.2016.03.002.

Guindon S, Dufayard JF, Lefort V, Anisimova M, Hordijk W, Gascuel O. 2010. New algorithms and methods to estimate maximum-likelihood phylogenies: Assessing the performance of PhyML 3.0. Systematic Biology 59:307-321. DOI: 10.1093/sysbio/syq010.

Guo Y, Tang K, Rowe LA, Li N, Roellig DM, Knipe K, Frace M, Yang C, Feng Y, Xiao L. 2015. Comparative genomic analysis reveals occurrence of genetic recombination in virulent Cryptosporidium hominis subtypes and telomeric gene duplications in Cryptosporidium parvum. BMC genomics 16:320. DOI: 10.1186/s12864-015-1517-1.

Hoang DT, Chernomor O, von Haeseler A, Minh BQ, Vinh LS. 2018. UFBoot2: Improving the Ultrafast Bootstrap Approximation. Molecular Biology and Evolution 35:518-522. DOI: 10.1093/molbev/msx281.

Holder A, Simon J, Strauser J, Taylor J, Shibberu Y. 2013. Dynamic Programming Used to Align Protein Structures with a Spectrum Is Robust. Biology 2:1296-1310. DOI: 10.3390/biology2041296.

Huerta-Cepas J, Forslund K, Coelho LP, Szklarczyk D, Jensen LJ, Von Mering C, Bork P. 2017. Fast genome-wide functional annotation through orthology assignment by eggNOG-mapper. Molecular

Peer) reviewing PDF | (2020:05:49002:1:1:NEW 8 Oct 2020) 
734

735

736

737

738

739

740

741

742

743

744

745

746

747

748

749

750

751

752

753

754

755

756

757

758

759

760

761

762

763

764

765

766

767

768

769

770

771

772

773

774

775

776

777

778

779

780

781

782

783

784

Biology and Evolution 34:2115-2122. DOI: 10.1093/molbev/msx148.

Huynh MH, Boulanger MJ, Carruthers VB. 2014. A conserved apicomplexan microneme protein contributes to Toxoplasma gondii invasion and virulence. Infection and Immunity 82:4358-4368. DOI: 10.1128/IAI.01877-14.

Ifeonu OO, Chibucos MC, Orvis J, Su Q, Elwin K, Guo F, Zhang H, Xiao L, Sun M, Chalmers RM, Fraser CM, Zhu G, Kissinger JC, Widmerg G, Silva JC. 2016. Annotated draft genome sequences of three species of Cryptosporidium: C. meleagridis isolate UKMEL1, C. baileyi isolate TAMU-09Q1, and C. hominis isolates TU502 2012 and UKH1. Pathogens and Disease. DOI: 10.1093/femspd/ftw080.

Isaza JP, Galvan AL, Polanco V, Huang B, Matveyev A V., Serrano MG, Manque P, Buck GA, Alzate JF. 2015. Revisiting the reference genomes of human pathogenic Cryptosporidium species: Reannotation of C. parvum Iowa and a new C. hominis reference. Scientific Reports 5. DOI: 10.1038/srep16324.

Jain BP, Pandey S. 2018. WD40 Repeat Proteins: Signalling Scaffold with Diverse Functions. Protein Journal 37:391-406. DOI: 10.1007/s10930-018-9785-7.

Ji D-D, Arnot DE. 1997. A Plasmodium falciparum homologue of the ATPase subunit of a multi- protein complex involved in chromatin remodelling for transcription. Molecular and Biochemical Parasitology 88:151-162. DOI: 10.1016/S0166-6851(97)00089-3.

Khan A, Shaik JS, Grigg ME. 2017. Genomics and molecular epidemiology of Cryptosporidium species. Acta Tropica:1-14. DOI: 10.1016/j.actatropica.2017.10.023.

Krogh A, Larsson B, Von Heijne G, Sonnhammer ELL. 2001. Predicting transmembrane protein topology with a hidden Markov model: Application to complete genomes. Journal of Molecular Biology 305:567-580. DOI: 10.1006/jmbi.2000.4315.

Lack JB, Reichard M V., Van Den Bussche RA. 2012. Phylogeny and evolution of the Piroplasmida as inferred from 18S rRNA sequences. International Journal for Parasitology 42:353-363. DOI: 10.1016/j.ijpara.2012.02.005.

Langer-Curry R, Riggs MW. 1999. Cryptosporidium parvum apical complex glycoprotein CSL contains a sporozoite ligand for intestinal epithelial cells. Infect. Immun. 67:5282-5291.

Li H, Durbin R. 2010. Fast and accurate long-read alignment with Burrows-Wheeler transform. Bioinformatics 26:589-595. DOI: 10.1093/bioinformatics/btp698.

Li H, Handsaker B, Wysoker A, Fennell T, Ruan J, Homer N, Marth G, Abecasis G, Durbin R. 2009. The Sequence Alignment/Map format and SAMtools. Bioinformatics 25:2078-2079. DOI: 10.1093/bioinformatics/btp352.

Li N, Xiao L, Cama VA, Ortega Y, Gilman RH, Guo M, Feng Y. 2013. Genetic recombination and Cryptosporidium hominis virulent subtype IbA10G2. Emerging Infectious Diseases 19:1573-1582. DOI: 10.3201/eid1910.121361.

Liu S, Roellig DM, Guo Y, Li N, Frace MA, Tang K, Zhang L, Feng Y, Xiao L. 2016. Evolution of mitosome metabolism and invasion-related proteins in Cryptosporidium. BMC Genomics 17:1-16. DOI: 10.1186/s12864-016-3343-5.

Magoc T, Salzberg SL. 2011. FLASH : fast length adjustment of short reads to improve genome assemblies Tanja Mago c. 27:2957-2963. DOI: 10.1093/bioinformatics/btr507.

De Maio N, Shaw LP, Hubbard A, George S, Sanderson ND, Swann J, Wick R, Oun MA, Stubberfield E, Hoosdally SJ, Crook DW, Peto TEA, Sheppard AE, Bailey MJ, Read DS, Anjum MF, Sarah Walker A, Stoesser N. 2019. Comparison of long-read sequencing technologies in the hybrid assembly of complex bacterial genomes. Microbial Genomics 5:1-21. DOI: 10.1099/mgen.0.000294.

Mazurie AJ, Alves JM, Ozaki LS, Zhou S, Schwartz DC, Buck GA. 2013a. Comparative genomics of cryptosporidium. International Journal of Genomics 2013. DOI: 10.1155/2013/832756.

Mazurie AJ, Alves JM, Ozaki LS, Zhou S, Schwartz DC, Buck GA. 2013b. Comparative Genomics of Cryptosporidium. Hindawi Publishing Corporation 2013:1-9. DOI: 10.1155/2013/832756.

Miles A, Iqbal Z, Vauterin P, Pearson R, Campino S, Theron M, Gould K, Mead D, Drury E, O’Brien J, Rubio VR, Macinnis B, Mwangi J, Samarakoon U, Ranford-Cartwright L, Ferdig M, Hayton K, Su

Peer) reviewing PDF | (2020:05:49002:1:1:NEW 8 Oct 2020) 
785

786

787

788

789

790

791

792

793

794

795

796

797

798

799

800

801

802

803

804

805

806

807

808

809

810

811

812

813

814

815

816

817

818

819

820

821

822

823

824

825

826

827

828

829

830

831

832

833

834

835
XZ, Wellems T, Rayner J, McVean G, Kwiatkowski D. 2016. Indels, structural variation, and recombination drive genomic diversity in Plasmodium falciparum. Genome Research 26:12881299. DOI: $10.1101 /$ gr.203711.115.

Morris A, Robinson G, Swain MT, Chalmers RM. 2019. Direct Sequencing of Cryptosporidium in Stool Samples for Public Health. Frontiers in Public Health 7. DOI: 10.3389/fpubh.2019.00360.

Nader JL, Mathers TC, Ward BJ, Pachebat JA, Swain MT, Robinson G, Chalmers RM, Hunter PR, van Oosterhout C, Tyler KM. 2019. Evolutionary genomics of anthroponosis in Cryptosporidium. Nature Microbiology 4:826-836. DOI: 10.1038/s41564-019-0377-x.

Nattestad M, Schatz MC. 2016. Assemblytics: A web analytics tool for the detection of variants from an assembly. Bioinformatics 32:3021-3023. DOI: 10.1093/bioinformatics/btw369.

Nickel W. 2003. The mystery of nonclassical protein secretion: A current view on cargo proteins and potential export routes. European Journal of Biochemistry 270:2109-2119. DOI: 10.1046/j.14321033.2003.03577.x.

Ogata H, Goto S, Sato K, Fujibuchi W, Bono H, Kanehisa M. 1999. KEGG: Kyoto encyclopedia of genes and genomes. Nucleic Acids Research 27:29-34. DOI: 10.1093/nar/27.1.29.

Okhuysen PC, Chappell CL. 2002. Cryptosporidium virulence determinants - Are we there yet? International Journal for Parasitology 32:517-525. DOI: 10.1016/S0020-7519(01)00356-3.

Pain A, Crossman L, Parkhill J. 2005. Comparative Apicomplexan genomics. Nature 3:454-455. DOI: 10.1038/nrmicro1164.

Pérez-Cordón G, Robinson G, Nader J, Chalmers RM. 2016. Discovery of new variable number tandem repeat loci in multiple Cryptosporidium parvum genomes for the surveillance and investigation of outbreaks of cryptosporidiosis. Experimental Parasitology 169:119-128. DOI: 10.1016/j.exppara.2016.08.003.

Puiu D. 2004. CryptoDB: the Cryptosporidium genome resource. Nucleic Acids Research 32:329D - 331. DOI: $10.1093 /$ nar/gkh050.

Ren X, Zhao J, Zhang L, Ning C, Jian F, Wang R, Lv C, Wang Q, Arrowood MJ, Xiao L. 2012. Cryptosporidium tyzzeri n. sp. (Apicomplexa: Cryptosporidiidae) in domestic mice (Mus musculus). Exp Parasitol. 130:274-81. DOI: 10.1016/j.exppara.2011.07.012.

Ryan U, Fayer R, Xiao L. 2014. Cryptosporidium species in humans and animals: current understanding and research needs. Parasitology 141:1667-85. DOI: 10.1017/S0031182014001085.

Ryan U, Paparini A, Monis P, Hijjawi N. 2016. It's official- Cryptosporidium is a gregarine: What are the implications for the water industry? Water Research 105:305-313. DOI: 10.1016/j.watres.2016.09.013.

Schwarz G. 1978. Estimating the Dimension of a Model. The Annals of Statistics 6:461-464. DOI: $10.1214 /$ aos/1176344136.

Shimodaira H, Hasegawa M. 1999. Multiple Comparisons of Log-Likelihoods with Applications to Phylogenetic Inference. Molecular Biology and Evolution 16:1114-1116. DOI: 10.1093/oxfordjournals.molbev.a026201.

Sikora P, Andersson S, Winiecka-Krusnell J, Hallström B, Alsmark C, Troell K, Beser J, Arrighi RBG. 2017a. Genomic variation in IbA10G2 and other patient-derived cryptosporidium hominis subtypes. Journal of Clinical Microbiology 55:844-858. DOI: 10.1128/JCM.01798-16.

Sikora P, Arrighi RBG, Beser J, Andersson S. 2017b. Genomic variation in IbA10G2 and other patientderived cryptosporidium hominis subtypes. Journal of Clinical Microbiology 55:844-858. DOI: http://dx.doi.org/10.1128/JCM.01798-16.

Šlapeta J. 2013. Cryptosporidiosis and Cryptosporidium species in animals and humans: A thirty colour rainbow? International Journal for Parasitology 43:957-970. DOI: 10.1016/j.ijpara.2013.07.005.

Sow SO, Muhsen K, Nasrin D, Blackwelder WC, Wu Y, Farag TH, Panchalingam S, Sur D, Zaidi AKM, Faruque ASG, Saha D, Adegbola R, Alonso PL, Breiman RF, Bassat Q, Tamboura B, Sanogo D, Onwuchekwa U, Manna B, Ramamurthy T, Kanungo S, Ahmed S, Qureshi S, Quadri F, Hossain A, Das SK, Antonio M, Hossain MJ, Mandomando I, Nhampossa T, Acácio S, Omore R, Oundo JO, Ochieng JB, Mintz ED, O’Reilly CE, Berkeley LY, Livio S, Tennant SM, Sommerfelt H, Nataro JP,

Peer) reviewing PDF | (2020:05:49002:1:1:NEW 8 Oct 2020) 
836

837

838

839

840

841

842

843

844

845

846

847

848

849

850

851

852

853

854

855

856

857

858

859

860

861

862

863

864

865

866

867

868

869

870

871

872

873

874

875

876

877

878

879

880

881

882

883

884

885

886
Ziv-Baran T, Robins-Browne RM, Mishcherkin V, Zhang J, Liu J, Houpt ER, Kotloff KL, Levine MM. 2016. The Burden of Cryptosporidium Diarrheal Disease among Children $<24$ Months of Age in Moderate/High Mortality Regions of Sub-Saharan Africa and South Asia, Utilizing Data from the Global Enteric Multicenter Study (GEMS). PLOS Neglected Tropical Diseases 10:e0004729. DOI: 10.1371/journal.pntd.0004729.

Stensvold CR, Beser J, Axen C, Lebbad M. 2014. High applicability of a novel method for gp60-based subtyping of Cryptosporidium meleagridis. Journal of Clinical Microbiology 52:2311-2319. DOI: 10.1128/JCM.00598-14.

Stirnimann CU, Petsalaki E, Russell RB, Christoph WM. 2010. WD40 proteins propel cellular networks. Review Trends in Biochemical Sciences Vol.35 35:531-538. DOI: 10.1016/j.tibs.2010.04.003.

Su J, Jin C, Wu H, Fei J, Li N, Guo Y, Feng Y, Xiao L. 2019. Differential expression of three cryptosporidium species-specific MEDLE proteins. Frontiers in Microbiology 10. DOI: 10.3389/fmicb.2019.01177.

Sulaiman IM, Lal AA, Xiao L. 2002. Molecular Phylogeny and Evolutionary Relationships of Cryptosporidium Parasites at the Actin Locus. The Journal of Parasitology 88:388. DOI: $10.2307 / 3285594$.

Sullivan WJ, Monroy MA, Bohne W, Nallani KC, Chrivia J, Yaciuk P, Smith CK, Queener SF. 2003. Molecular cloning and characterization of an SRCAP chromatin remodeling homologue in Toxoplasma gondii. Parasitology Research 90:1-8. DOI: 10.1007/s00436-002-0814-1.

Sullivan WJ, Radke JB, Kim K, White MW. 2013. Epigenetic and Genetic Factors that Regulate Gene Expression in Toxoplasma gondii. Elsevier. DOI: 10.1016/B978-0-12-396481-6.00018-0.

Templeton TJ, Iyer LM, Anantharaman V, Enomoto S, Abrahante JE, Subramanian GM, Hoffman SL, Abrahamsen MS, Aravind L. 2004. Comparative analysis of apicomplexa and genomic diversity in eukaryotes. Genome Research 14:1686-1695. DOI: 10.1101/gr.2615304.

Theys K, Lemey P, Vandamme AM, Baele G. 2019. Advances in Visualization Tools for Phylogenomic and Phylodynamic Studies of Viral Diseases. Frontiers in Public Health 7. DOI: 10.3389/fpubh.2019.00208.

Trifinopoulos J, Nguyen LT, von Haeseler A, Minh BQ. 2016. W-IQ-TREE: a fast online phylogenetic tool for maximum likelihood analysis. Nucleic acids research 44:W232-W235. DOI: 10.1093/nar/gkw256.

Troeger C, Forouzanfar M, Rao PC, Khalil I, Brown A, Reiner RC, Fullman N, Thompson RL, Abajobir A, Ahmed M, Alemayohu MA, Alvis-Guzman N, Amare AT, Antonio CA, Asayesh H, Avokpaho E, Awasthi A, Bacha U, Barac A, Betsue BD, Beyene AS, Boneya DJ, Malta DC, Dandona L, Dandona R, Dubey M, Eshrati B, Fitchett JRA, Gebrehiwot TT, Hailu GB, Horino M, Hotez PJ, Jibat T, Jonas JB, Kasaeian A, Kissoon N, Kotloff K, Koyanagi A, Kumar GA, Rai RK, Lal A, El Razek HMA, Mengistie MA, Moe C, Patton G, Platts-Mills JA, Qorbani M, Ram U, Roba HS, Sanabria J, Sartorius B, Sawhney M, Shigematsu M, Sreeramareddy C, Swaminathan S, Tedla BA, Jagiellonian RTM, Ukwaja K, Werdecker A, Widdowson MA, Yonemoto N, El Sayed Zaki M, Lim SS, Naghavi M, Vos T, Hay SI, Murray CJL, Mokdad AH. 2017. Estimates of global, regional, and national morbidity, mortality, and aetiologies of diarrhoeal diseases: a systematic analysis for the Global Burden of Disease Study 2015. The Lancet Infectious Diseases 17:909-948. DOI: 10.1016/S1473-3099(17)30276-1.

Vaser R, Adusumalli S, Leng SN, Sikic M, Ng P. 2016. SIFT missense predictions for genomes. Nat. Protoc 11:1073-1081. DOI: doi:10.1038/nprot.2015.123.

Wagnerová P, Sak B, McEvoy J, Rost M, Perec Matysiak A, Ježková J, Kváč M. 2015. Genetic diversity of Cryptosporidium spp. including novel identification of the Cryptosporidium muris and Cryptosporidium tyzzeri in horses in the Czech Republic and Poland. Parasitology Research 114:1619-1624. DOI: 10.1007/s00436-015-4353-y.

Watson M. 2018.A simple test for uncorrected insertions and deletions (indels) in bacterial genomes Weir W, Karagenç T, Gharbi M, Simuunza M, Aypak S, Aysul N, Darghouth MA, Shiels B, Tait A. 2011. Population diversity and multiplicity of infection in Theileria annulata. International Journal 
887

888

889

890

891

892

893

894

895

896

897

898

899

900

901

902

903

904

905

906

907

908

909

910

911

912

913

914

915

916

917

918

919

920

921

for Parasitology 41:193-203. DOI: 10.1016/j.ijpara.2010.08.004.

Widmer G. 2018. Diverse single-amino-acid repeat profiles in the genus Cryptosporidium. Mineralogical Magazine 145:1151-1160. DOI: 10.1017/S0031182018000112.

Widmer G, Lee Y, Hunt P, Martinelli A, Tolkoff M, Bodi K. 2012. Comparative genome analysis of two Cryptosporidium parvum isolates with different host range. Infection, Genetics and Evolution 12:1213-1221. DOI: 10.1016/j.meegid.2012.03.027.

Widmer G, Sullivan S. 2012. Genomics and population biology of Cryptosporidium species. Parasite Immunology 34:61-71. DOI: 10.1111/j.1365-3024.2011.01301.x.

Xiao L. 2010. Molecular epidemiology of cryptosporidiosis: An update. Experimental Parasitology 124:80-89. DOI: 10.1016/j.exppara.2009.03.018

Xiao L, Feng Y. 2017. Molecular epidemiologic tools for waterborne pathogens Cryptosporidium spp. and Giardia duodenalis. Food and Waterborne Parasitology 8-9:14-32. DOI: 10.1016/j.fawpar.2017.09.002.

Xu Z, Guo Y, Roellig DM, Feng Y, Xiao L. 2019. Comparative analysis reveals conservation in genome organization among intestinal Cryptosporidium species and sequence divergence in potential secreted pathogenesis determinants among major human-infecting species. BMC Genomics 20. DOI: 10.1186/s12864-019-5788-9.

Xu C, Min J. 2011. Structure and function of WD40 domain proteins. Protein and Cell 2:202-214. DOI: 10.1007/s13238-011-1018-1.

Xu P, Widmer G, Wang Y, Ozaki LS, Alves JM, Serrano MG, Puiu D, Manque P, Akiyoshi D, Mackey AJ, Pearson WR, Dear PH, Bankier AT, Peterson DL, Abrahamsen MS, Kapur V, Tzipori S, Buck GA. 2004. The Genome of Cryptosporidium hominis. 431. DOI: 10.1038/nature02990.

Ye J, Zhang Y, Cui H, Liu J, Wu Y, Cheng Y, Xu H, Huang X, Li S, Zhou A, Zhang X, Bolund L, Chen Q, Wang J, Yang H, Fang L, Shi C. 2018. WEGO 2.0: A web tool for analyzing and plotting GO annotations, 2018 update. Nucleic Acids Research 46:W71-W75. DOI: 10.1093/nar/gky400.

Young AD, Gillung JP. 2020. Phylogenomics - principles, opportunities and pitfalls of big-data phylogenetics. Systematic Entomology. DOI: 10.1111/syen.12406.

Zahedi A, Gofton AW, Jian F, Paparini A, Oskam C, Ball A, Robertson I, Ryan U, Blunt DS, Khramtsov N V, Upton SJ, Montelone B a, Isaza JP, Galvan AL, Polanco V, Huang B, Matveyev A V., Serrano MG, Manque P, Buck GA, Alzate JF, Of PB, Guo Y, Tang K, Rowe LA, Li N, Roellig DM, Knipe K, Frace MA, Yang C, Feng Y, Xiao L, Liu S, Roellig DM, Guo Y, Li N, Frace MA, Tang K, Zhang L, Feng Y, Xiao L, Bouzid M, Hunter PR, Chalmers RM, Tyler KM. 2013. Comparative genomic analysis reveals occurrence of genetic recombination in virulent Cryptosporidium hominis subtypes and telomeric gene duplications in Cryptosporidium parvum. BMC Genomics 5:1-14. DOI: $10.1186 / \mathrm{s} 12864-015-1517-1$. 
Figure 1

Accumulation of SNVs in Cryptosporidium species.

Box plots of the Cryptosporidium species analyzed and the number of total SNVs detected.

The medians of the SNVs found in each species are (A) C. meleagridis $(624,607),(B)$ C. hominis $(223,640)$, and $(\mathbf{C})$ C. parvum $(2,116)$. Outliers in C. meleagridis and C. hominis correspond to the UKMEL3, SWEH2, and SWEH5, respectively. Concerning C. parvum, outliers correspond to anthroponotic UKP14 and UKP15 isolates.

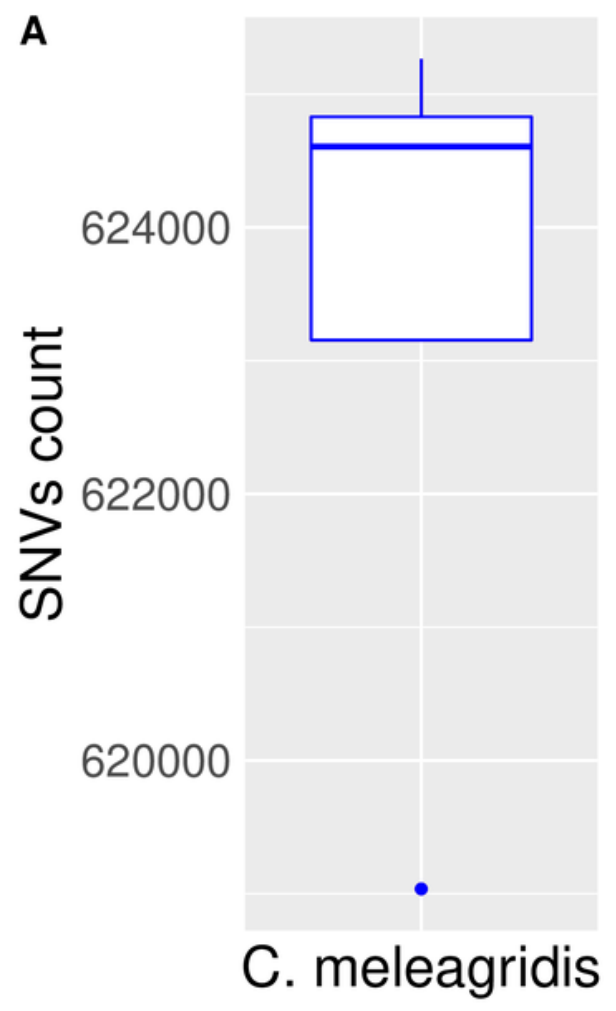

B

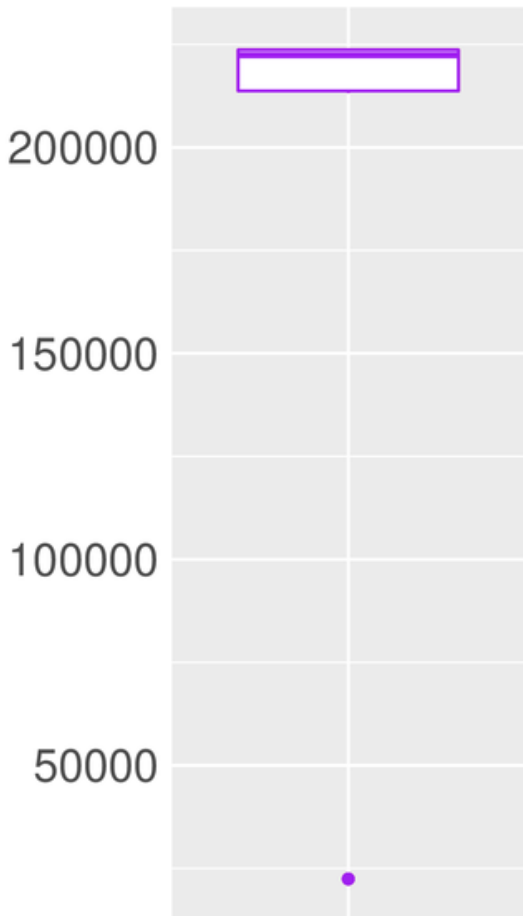

C. hominis
C 20000

15000

10000

5000

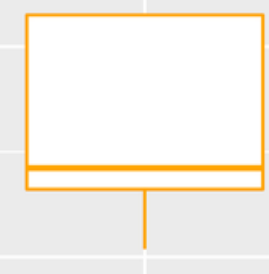

C. parvum 


\section{Figure 2}

Phylogenomic analysis of Cryptosporidium species.

Maximum Likelihood (ML) tree was based on the SNVs present within the 24 Cryptosporidium genomes studied. The presented tree is unrooted, and the longest branch was selected as an arbitrary outgroup. The horizontal scale line represents the number of base substitutions per site analyzed. TVM was the evolutionary model. The supports of the branches are based on an analysis of 1000 aLRT replications (\%) / ultra-fast bootstrap replications (\%). Red branches have an aLRT and bootstrap value of $100 \%$. Branches are labeled with the isolate ID and allelic family based on the gp60 gene. 


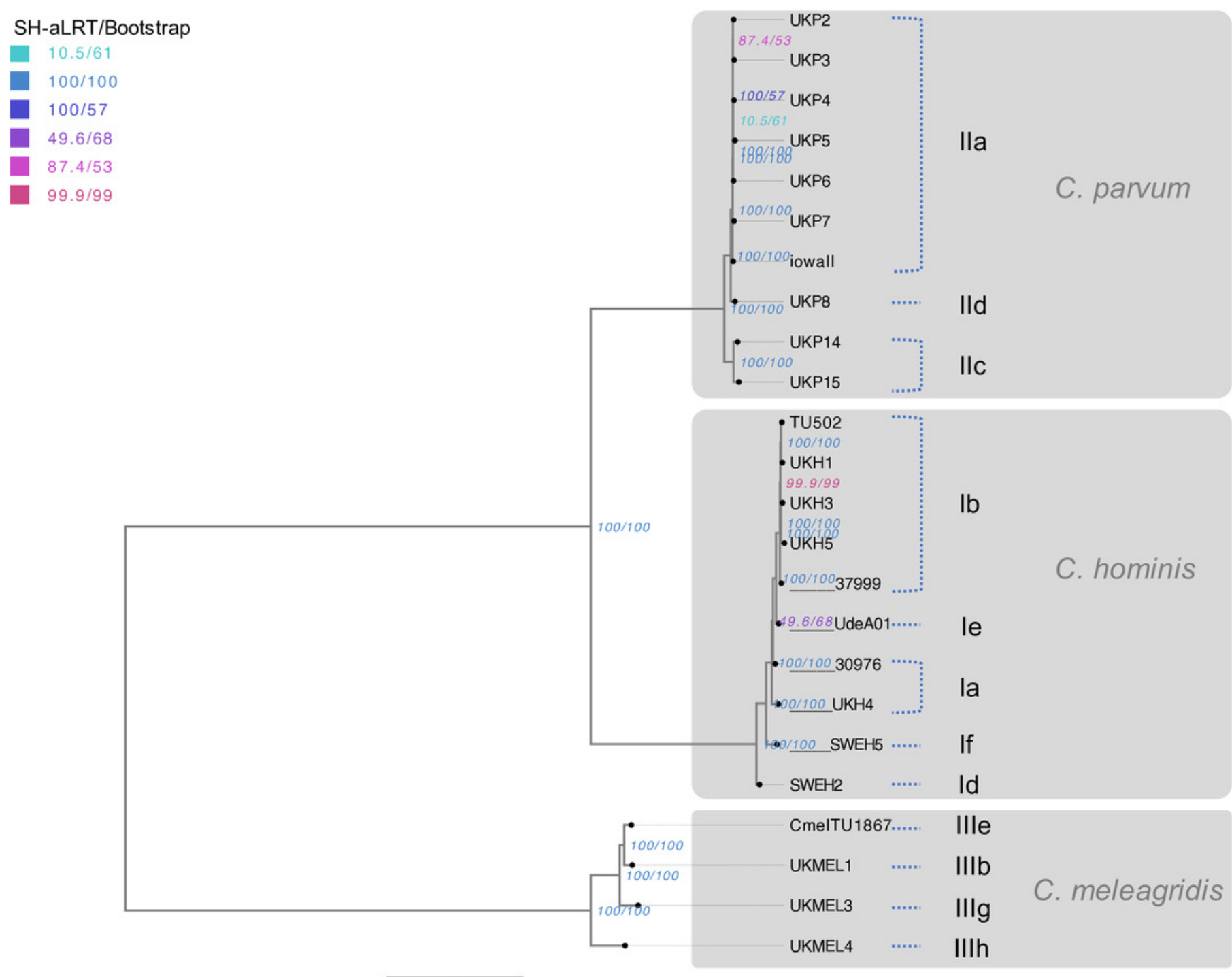




\section{Figure 3}

Genes detected with deleterious mutations in the three Cryptosporidium species.

Venn diagram showing the genes with shared and exclusive deleterious mutations in the three Cryptosporidium species included in the study.

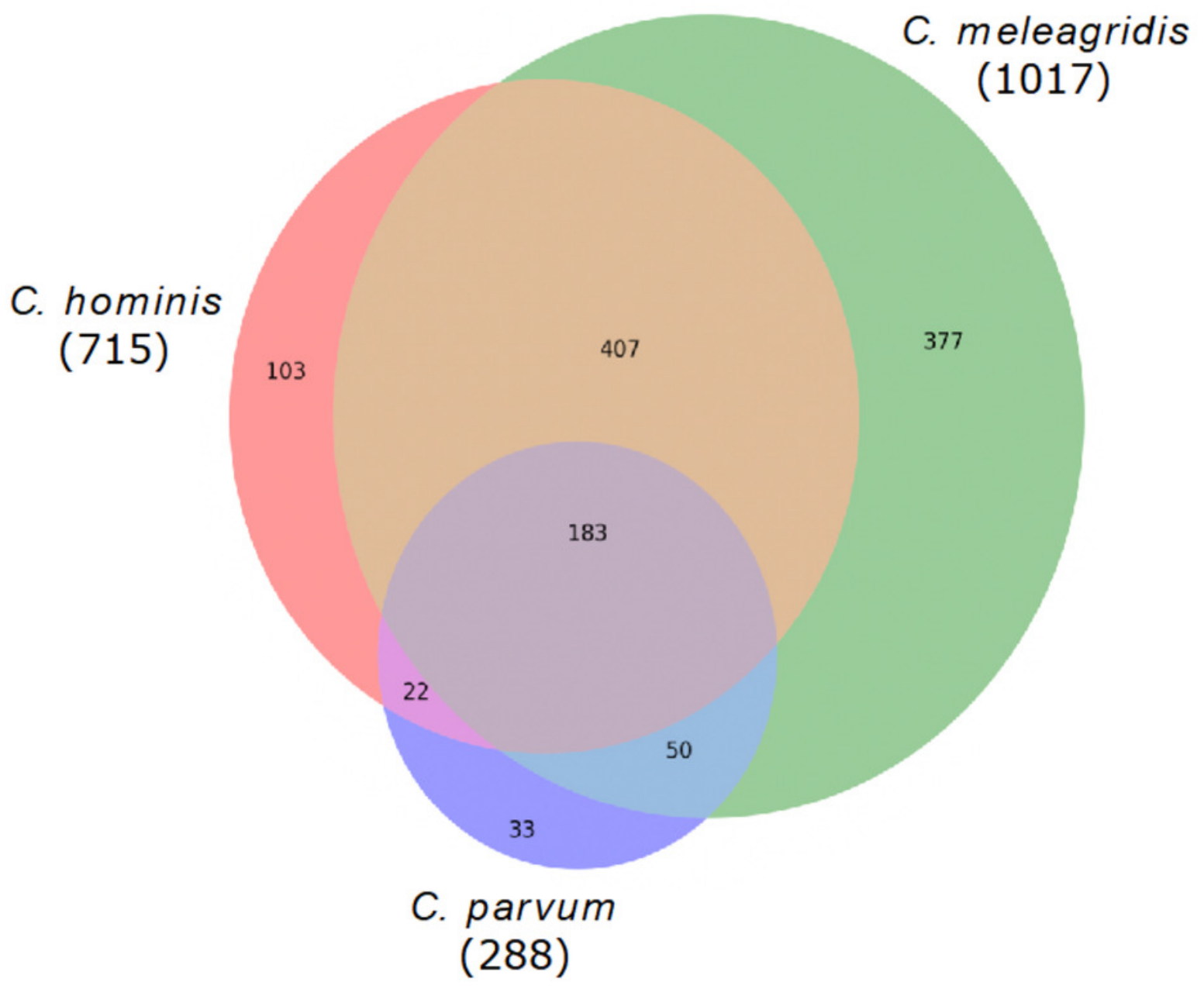


Figure 4

KEGG orthology analysis of genes with deleterious mutations shared by the three Cryptosporidium species.

Assignment of $\mathrm{KO}$ terms to genes with deleterious mutations detected in the three

Cryptosporidium species through the KAAS server in the KEGG database.

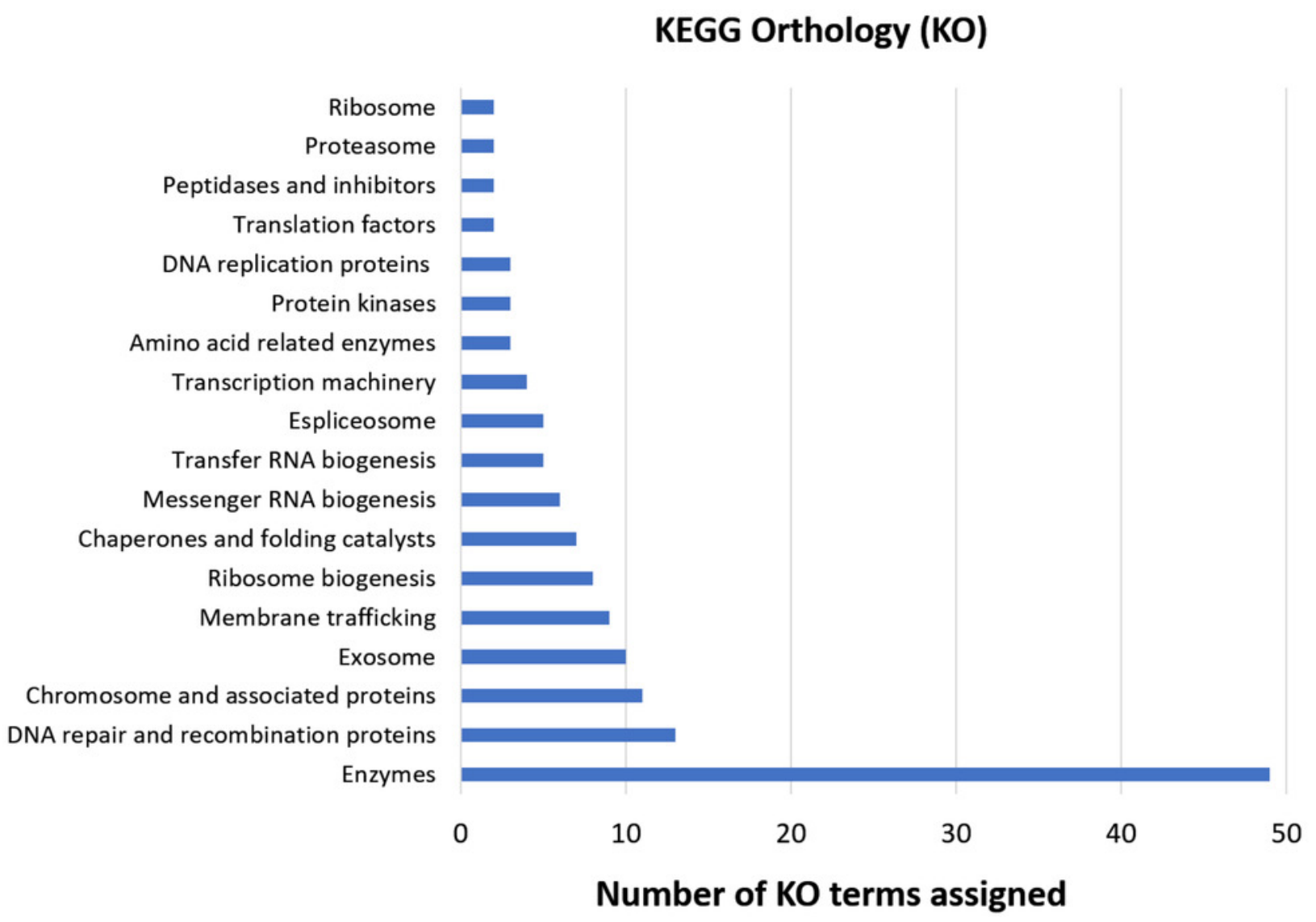


Figure 5

KEGG orthology analysis of genes with species-exclusive deleterious mutations.

Assignment of KO terms to genes with species-exclusive deleterious mutations via the KAAS

server in the KEGG database in C. hominis (A), C.parvum (B), and C. meleagridis (C). 


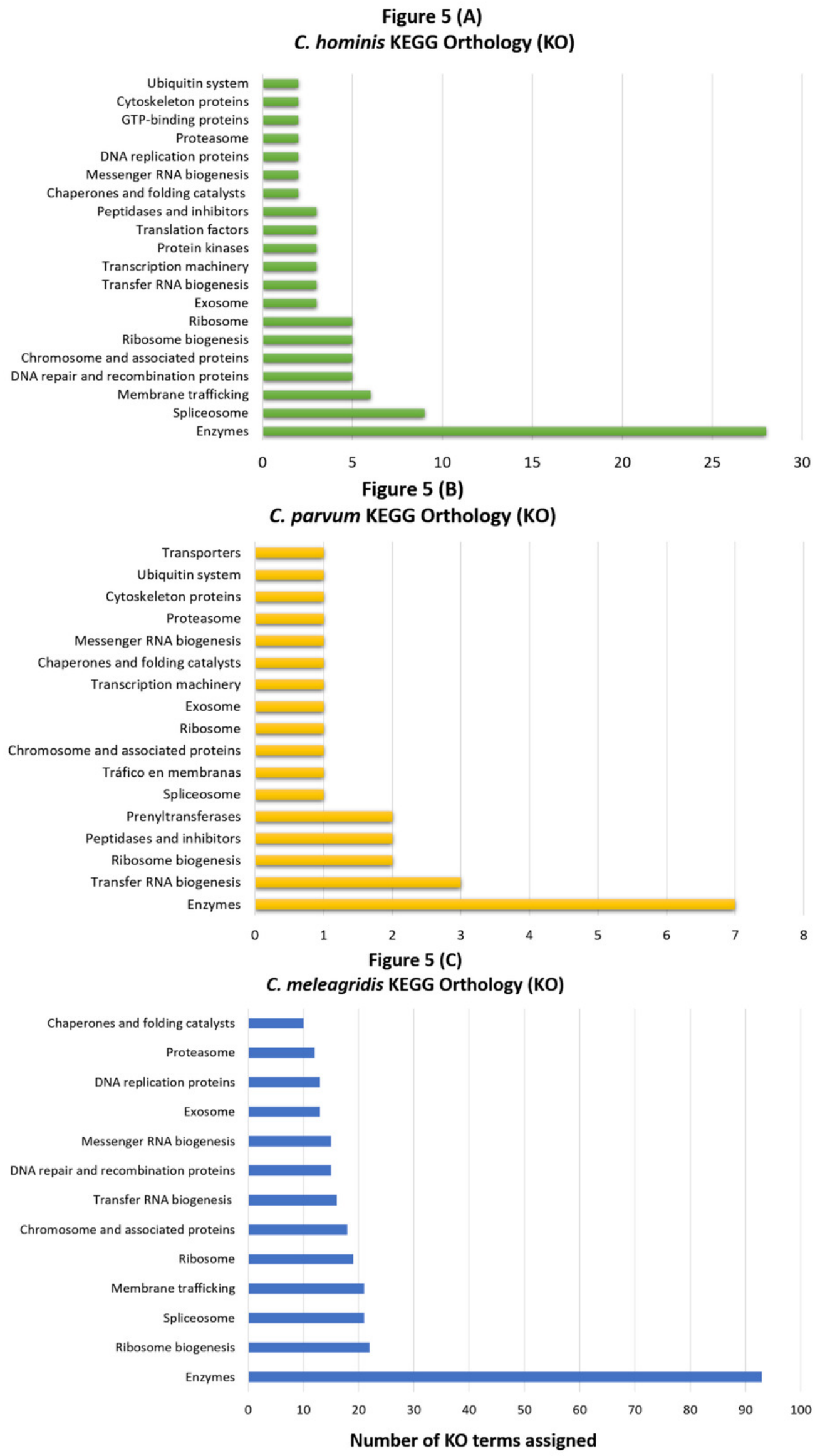

Peer] reviewing PDF | (2020:05:49002:1:1:NEW 8 Oct 2020) 


\section{Figure 6}

Analysis of genes with indel events in the three Cryptosporidium species.

Venn diagram that shows the genes with indel events that are shared and exclusive in the three Cryptosporidium species.

\section{C. meleagridis}

\section{C. hominis}

14

27

62

C. parvum 